\title{
Organocatalytic Domino Michael-Knoevenagel Condensation Reaction for the Synthesis of Optically Active 3-Diethoxyphosphoryl-2-oxocyclohex-3-enecarboxylates
}

\author{
Eukasz Albrecht, ${ }^{[a, b]}$ Bo Richter, ${ }^{[a]}$ Carlos Vila, ${ }^{[a]}$ Henryk Krawczyk, ${ }^{[b]}$ and \\ Karl Anker Jørgensen*[a]
}

\begin{abstract}
A novel, organocatalytic, highly enantio- and diastereoselective synthetic approach towards optically active 6-substituted-3-diethoxyphosphoryl-2-oxocyclohex-3-enecarboxylates is presented. Our methodology utilizes a Michael-Knoevenagel domino reaction sequence of ethyl 4-diethoxyphosphoryl-3-oxobutanoate and $\alpha, \beta$-unsaturated aldehydes catalyzed by a chiral diarylprolinol ether. The cyclohexenecarboxylates obtained are particularly well suited for the preparation of highly functionalized cyclohexene and cyclohexane derivatives, with up to four chiral centers and high levels of stereocontrol.
\end{abstract}

Keywords: asymmetric synthesis . domino reactions - Michael-Knoevenagel reactions $\cdot$ organocatalysis • phosphonates

\section{Introduction}

Continuously growing interest in the chemistry of natural products and other molecules of general importance such as pharmaceuticals, chiral building blocks, and agromaterials has resulted in development of a great number of synthetic approaches towards these types of compounds, based on stereodifferentiating reactions by using inductors of chirality in sub-stochiometric amounts. In recent years, asymmetric organocatalysis has emerged as a very attractive method for the construction of enantiomerically pure and enriched compounds, being complementary to metal and enzyme catalysis. ${ }^{[1]}$ In particular, a number of highly enantioselective transformations of carbonyl substrates catalyzed by chiral secondary amines involving the corresponding enamine ${ }^{[2]}$ or iminium $^{[3]}$ intermediates have been successfully performed.

Domino reactions, in which more than one bond is being formed in a multistep one-pot reaction sequence, giving

[a] Ł. Albrecht, B. Richter, C. Vila, Prof. Dr. K. A. Jørgensen Department of Chemistry

Danish National Research Foundation: Centre for Catalysis

Aarhus University 8000 Aarhus C (Denmark)

Fax: (+45) 8919-6199

E-mail: kaj@chem.au.dk

[b] Ł. Albrecht, Prof. Dr. H. Krawczyk

Department of Chemistry, Institute of Organic Chemistry

Technical University of Lodz, Żeromskiego 116

90-924 Lodz (Poland) access to molecules of complex architecture without isolation and purification of the intermediates, are of great importance in organic chemistry. As it has been demonstrated, organocatalysis provides a possibility to perform such reactions in an asymmetric fashion with high levels of stereocontrol. The immense progress in this field of organocatalysis has been recently reviewed. ${ }^{[4]}$ In our efforts to contribute to this extensively developing research area, we have recently established several multicomponent domino processes, demonstrating that even up to five stereocenters ${ }^{[5 h]}$ can be formed in an organocatalytic domino reaction with excellent enantio- and diastereoselectivity. ${ }^{[5]}$ Synthetic utility of organocatalytic domino processes in which the first step consists of an iminium-catalyzed Michael reaction for the synthesis of optically active carbo- and heterocyclic products is well recognized. ${ }^{[5,6]}$ However, to the best of our knowledge, the cascade reaction proceeding as a Michael-Knoevenagel condensation sequence is essentially unknown.

Chiral, differently substituted, 2-oxocyclohex-3-enecarboxylates constitute an important group of organic molecules that has found many applications as useful building blocks in organic synthesis. ${ }^{[7]}$ As a consequence, a number of preparative routes to these compounds has been developed. ${ }^{[7,8]}$ One of the most effective and operationally simple is that based on the $[3+3]$ annulation reaction. ${ }^{[7,8 b-d]}$ The synthesis of optically active 3-diethoxyphosphoryl-2-oxocyclohex-3-enecarboxylates has not been previously described in the literature. However, this class of phosphonates is par- 
ticularly interesting due to the their potential synthetic utility.

Therefore, we report an organocatalytic, diastereo- and enantioselective Michael-Knoevenagel domino one-pot approach towards highly functionalized 6-substituted-3-diethoxyphosphoryl-2-oxocyclohex-3-enecarboxylates (3). We envisioned the reaction sequence involving Michael addition of ethyl 4-diethoxyphosphoryl-3-oxobutanoate (1) to $\alpha, \beta$-unsaturated aldehydes (2) by using iminium activation, followed by subsequent Knoevenagel condensation of the resulting adducts as a facile and efficient approach to the target compounds (Scheme 1). Our methodology benefits

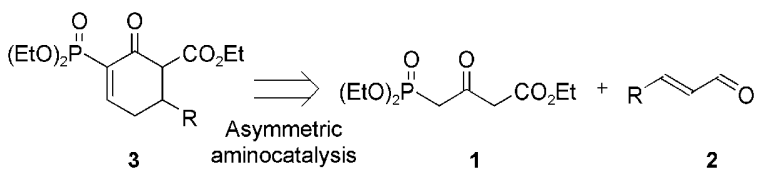

Scheme 1. Enantioselective organocatalytic approach towards 6-substituted-3-diethoxyphosphoryl-2-oxocyclohex-3-enecarboxylates 3 .

from operational simplicity and benign reaction conditions. Moreover, we present that these optically active products are useful starting materials for further diastereoselective transformations leading to the formation of various enantiomerically enriched cyclohexene and cyclohexane derivatives with up to four stereocenters. Therefore, the optically active products obtained, can be considered as versatile building blocks for the synthesis of molecules of higher complexity.

\section{Results and Discussion}

Screening and scope: At the outset of our studies, investigations were carried out in order to find the optimal reaction conditions for the formation of enantiomerically enriched 6substituted-3-diethoxyphosphoryl-2-oxocyclohex-3-enecarboxylates 3 (Table 1 ). We anticipated that 2-[bis(3,5-bistrifluoromethylphenyl)trimethyl-silylanyloxymethyl]pyrrolidine 4 should be particularly well suited as a catalyst of our envisioned domino reaction, since its general catalytic activity has been confirmed in numerous aminocatalytic transformations. ${ }^{[9]}$

Cinnamaldehyde $\mathbf{2 a}$ was chosen as a model substrate. To our delight its reaction with ethyl 4-diethoxyphosphoryl-3oxobutanoate 1 in $\mathrm{CH}_{2} \mathrm{Cl}_{2}$ in the presence of $4(10 \mathrm{~mol} \%)$ was found to take place efficiently at room temperature and was completed within $24 \mathrm{~h}$ (Table 1 , entry 1 ). We also observed that the originally formed Michael adduct underwent, under these conditions, spontaneous intramolecular Knoevenagel reaction to give cyclohex-2-enecarboxylate $\mathbf{3 a}$ with high enantioselectivity (94\% enantiomeric excess $(e e)$ ) and excellent diasteroselectivity (>95:5). In the next stage of the studies, different solvents, concentrations, and temperatures were screened. The Michael-Knoevenagel domino reaction could be efficiently performed in all solvents tested
Table 1. Screening of the reaction conditions of cinnamaldehyde $\mathbf{2} \mathbf{a}$ with ethyl 4-diethoxyphosphoryl-3-oxobutanoate 1 catalyzed by 4. $^{\text {[a] }}$

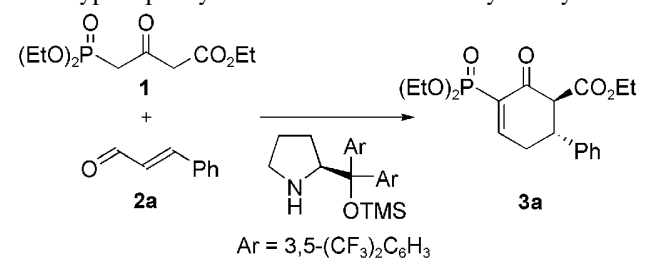

4

\begin{tabular}{lllllll}
\hline & Solvent & Conc. [M] & $T\left[{ }^{\circ} \mathrm{C}\right]$ & Yield [\%] & $e e[\%]^{[\mathrm{b}]}$ & $\mathrm{dr}^{[\mathrm{c}]}$ \\
\hline 1 & $\mathrm{CH}_{2} \mathrm{Cl}_{2}$ & 0.1 & RT & 66 & 94 & $>95: 5$ \\
2 & $\mathrm{CHCl}_{3}$ & 0.1 & RT & 67 & 94 & $>95: 5$ \\
3 & EtOH & 0.1 & RT & 55 & 96 & $>95: 5$ \\
4 & toluene & 0.1 & RT & 42 & 94 & $>95: 5$ \\
5 & $\mathrm{CH}_{2} \mathrm{Cl}_{2}$ & 0.2 & RT & 68 & 97 & $>95: 5$ \\
6 & $\mathrm{CH}_{2} \mathrm{Cl}_{2}$ & 0.5 & RT & 51 & 98 & $>95: 5$ \\
7 & $\mathrm{CH}_{2} \mathrm{Cl}_{2}$ & 0.2 & 0 & 65 & 98 & $>95: 5$ \\
$8^{[\mathrm{d}]}$ & $\mathrm{CH}_{2} \mathrm{Cl}_{2}$ & 0.2 & -30 & 72 & 98 & $>95: 5$ \\
$9^{[\mathrm{e}]}$ & $\mathrm{CH}_{2} \mathrm{Cl}_{2}$ & 0.2 & -30 & 76 & 98 & $>95: 5$ \\
\hline
\end{tabular}

[a] All reactions were performed at $0.125 \mathrm{mmol}$ scale with two equivalents of the aldehyde in the presence of $4(10 \mathrm{~mol} \%)$ for $24 \mathrm{~h}$. [b] Determined by HPLC on a chiral stationary phase. [c] Determined by ${ }^{31} \mathrm{P}$ NMR spectroscopy. [d] Reaction conducted for $48 \mathrm{~h}$. [e] Reaction performed with $\mathrm{PhCO}_{2} \mathrm{H}(10 \mathrm{~mol} \%)$ as co-catalyst.

affording 3a with high levels of stereocontrol (entries 1-4). However, chlorinated solvents were the most useful in terms of yields (Table 1 , entries 1 and 2). $\mathrm{CH}_{2} \mathrm{Cl}_{2}$ turned out to be the solvent of choice and was used for concentration screening. Those studies revealed that enantioselectivity of the reaction was improved with increasing concentration of phosphonate 1 (Table 1, entries 5, 6). Finally, the temperature effect was evaluated (Table 1, entries 7-9) and the key parameters of the reaction were found (Table 1, entry 9). Performing the reaction at $-30^{\circ} \mathrm{C}$ in the presence of $\mathrm{PhCO}_{2} \mathrm{H}$ $(10 \mathrm{~mol} \%)$ as co-catalyst allowed us to obtain $\mathbf{3 a}$ in $76 \%$ yield, and with excellent enantio- and diastereoselectivity within $24 \mathrm{~h}$.

With the optimized conditions in hand, we investigated the scope of the reaction. The results are summarized in Table 2. Various substituted cinnamaldehydes $\mathbf{2 b - f}$ were treated with ethyl 4-diethoxyphosphoryl-3-oxobutanoate (1). The reaction proved to be general, since good yields (76$95 \%$ ), high diastereoselectivities (ranging from 87:13 to $>95: 5$ ), and excellent enantioselectivities (97-98\% ee) were observed in all the cases. The position of the substituent on the aromatic ring had no significant influence on the stereochemical outcome of the reaction. Moreover, the MichaelKnoevenagel domino reaction with cinnamaldehydes bearing either electron-withdrawing or electron-donating substituents proceeded without noticeable changes in yield or stereoselectivity. The use of heteroaromatic 2-furyl substituted aldehyde $\mathbf{2} \mathbf{g}$ is also worth mentioning, leading to the formation of $\mathbf{3 g}$ with $71 \%$ yield, 9:1 dr and 97\% ee (Table 2, entry 7). As expected, the opposite enantiomer of the catalyst $(R)-\mathbf{4}$ gave access to the enantiomeric product ent-3a as a single diastereoisomer with $98 \%$ ee (Table 2, entry 8 ). 
Table 2. 2-[Bis(3,5-bistrifluoromethylphenyl)trimethylsilylanyloxymethyl]pyrrolidine-catalyzed reaction of aromatic aldehydes $\mathbf{2 a - g}$ with ethyl 4-diethoxyphosphoryl-3-oxobutanoate (1). ${ }^{[\mathrm{a}]}$

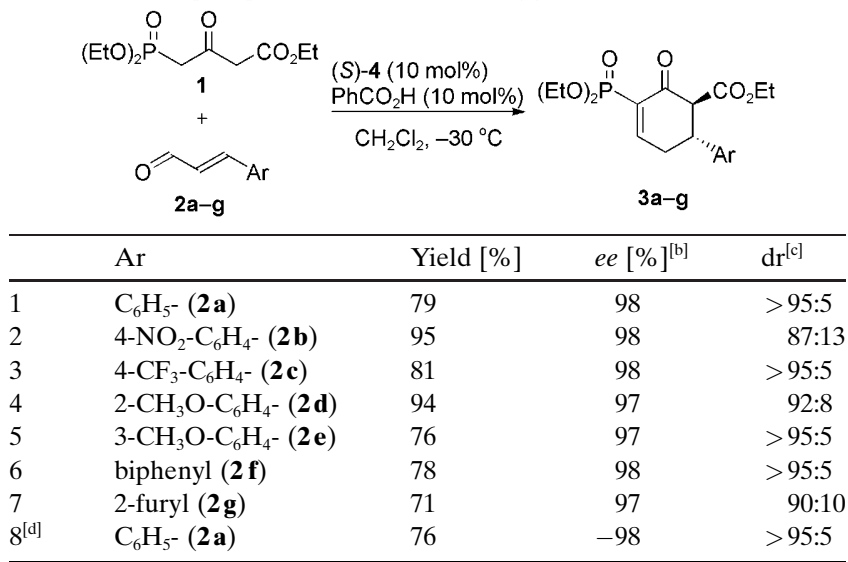

[a] All reactions were performed at $0.25 \mathrm{mmol}$ scale with two equivalents of the aldehyde in $\mathrm{CH}_{2} \mathrm{Cl}_{2}(1.25 \mathrm{~mL})$ for $24 \mathrm{~h}$. [b] Determined by HPLC on a chiral stationary phase. [c] Determined by ${ }^{31} \mathrm{P}$ NMR spectroscopy. [d] Reaction performed with enantiomeric catalyst $(R)-4$.

To our surprise, aliphatic enals turned out to be unreactive under the applied conditions. Therefore, a screening of a model reaction between 2-pentenal $(\mathbf{2} \mathbf{h})$ and compound $\mathbf{1}$ had to be performed and different additives were evaluated (Table 3). It became evident that the use of an additional basic co-catalyst, to facilitate the formation of the corre-

Table 3. Screening of the reaction conditions of 2-pentenal $(\mathbf{2} \mathbf{h})$ with ethyl 4-diethoxyphosphoryl-3-oxobutanoate (1) catalyzed by $4^{[\mathrm{a}]}$<smiles>CCOC(=O)CC(=O)CP(=O)(CC(=O)OCC)OCC</smiles>

$$
\begin{aligned}
& \mathrm{Ah} \\
& \mathrm{Ar}=3,5-\left(\mathrm{CF}_{3}\right)_{2} \mathrm{C}_{6} \mathrm{H}_{3}^{-}
\end{aligned}
$$

\begin{tabular}{|c|c|c|c|c|c|c|c|}
\hline & $\begin{array}{l}\text { Catalyst } \\
{[\mathrm{mol} \%]}\end{array}$ & $\begin{array}{l}\text { Additive } \\
{[\mathrm{mol} \%]}\end{array}$ & $\begin{array}{l}\text { Conv. } \\
{[\%]^{[\mathrm{b}]}}\end{array}$ & $\begin{array}{l}T \\
{\left[{ }^{\circ} \mathrm{C}\right]}\end{array}$ & $\begin{array}{l}\text { Yield } \\
{[\%]}\end{array}$ & $\begin{array}{l}e e \\
{[\%]^{[\mathrm{c}]}}\end{array}$ & $\mathrm{dr}^{[\mathrm{d}]}$ \\
\hline 1 & $S(20)$ & - & 0 & RT & nd & nd & nd \\
\hline 2 & $S(20)$ & $\mathrm{PhCO}_{2} \mathrm{H}(10)$ & 7 & RT & nd & nd & nd \\
\hline 3 & $S(20)$ & KF (10) & 55 & RT & nd & nd & nd \\
\hline 4 & $S(20)$ & $\mathrm{K}_{2} \mathrm{CO}_{3}(10)$ & 55 & RT & nd & nd & nd \\
\hline 5 & $S(20)$ & DABCO (10) & 89 & RT & 61 & 90 & $4: 1$ \\
\hline 6 & $S(20)$ & DHQ (10) & 100 & RT & 56 & 94 & $4.5: 1$ \\
\hline 7 & $S(10)$ & DHQ (5) & 93 & RT & 63 & 94 & 4.8:1 \\
\hline 8 & $R(10)$ & DHQ (5) & 86 & RT & 65 & -94 & $4: 1$ \\
\hline $9^{[\mathrm{e}]}$ & $S(10)$ & DHQ (20) & 94 & RT & 41 & 85 & $5.2: 1$ \\
\hline $10^{[\mathrm{f}]}$ & $S(10)$ & DHQ (5) & 100 & 0 & 56 & 93 & $4.4: 1$ \\
\hline $11^{[\mathrm{f}, \mathrm{g}]}$ & $S(10)$ & DHQ (5) & 67 & -30 & 60 & 97 & $6.5: 1$ \\
\hline $12^{[\mathrm{f}, \mathrm{h}]}$ & $S(10)$ & DHQ (5) & 100 & -30 & 74 & 96 & $4: 1$ \\
\hline
\end{tabular}

4

[a] All reactions were performed at $0.125 \mathrm{mmol}$ scale with two equivalents of the aldehyde in $\mathrm{CH}_{2} \mathrm{Cl}_{2}(1.25 \mathrm{~mL})$ for $24 \mathrm{~h}$. [b] Estimated after $24 \mathrm{~h}$ by ${ }^{31} \mathrm{P}$ NMR spectroscopy of the crude reaction mixture. [c] Determined by HPLC on a chiral stationary phase. [d] Determined by ${ }^{31} \mathrm{P}$ NMR spectroscopy. [e] Reaction conducted for $8 \mathrm{~d}$. [f] Concentration $0.2 \mathrm{M}$. [g] Reaction conducted for $48 \mathrm{~h}$. [h] Reaction performed in the presence of $\mathrm{PhCO}_{2} \mathrm{H}(2.5 \mathrm{~mol} \%)$ as co-catalyst. sponding enol, is crucial for the reaction to proceed in a desired manner (Table 3, entries 3-6). 1,4-Diazabicyclo[2.2.2] octane (DABCO) was found to be the best achiral basic additive, affording the Michael-Knoevenagel domino product $\mathbf{3 h}$ efficiently with $90 \%$ ee and 4:1 diastereomeric ratio (Table 3 , entry 5 ). In the next stage of screening, we decided to evaluate the influence of a chiral, basic co-catalyst on the reaction outcome. The use of hydroquinine (DHQ) as a chiral Brønsted base slightly increased enantioselectivity of the reaction (94\% ee, Table 3, entry 6). Further screening revealed that loading of both catalyst and co-catalyst could easily be lowered to 10 and $5 \mathrm{~mol} \%$, respectively, without diminishing neither yield, nor stereoselectivity (Table 3, entry 7). Moreover, the presence of the chiral cocatalyst had no major impact on the stereochemical outcome of the reaction, indicating that no matching-mismatching interactions between catalyst and co-catalyst occur. The absolute configuration of the product is determined only by the chirality of the catalyst 4 (compare entries 7 and 8). It is also worth noting that the relative ratio of catalyst and cocatalyst had appreciable influence on the results obtained. Increase of DHQ loading to $20 \mathrm{~mol} \%$, while maintaining catalyst loading at $10 \mathrm{~mol} \%$, significantly suppressed the reaction rate ( 8 days were required for full conversion of the starting phosphonate) leading to the formation of the product with lower yield and enantioselectivity (Table 3, entry 9). Further experiments revealed that the enantioselectivity of the reaction is slightly temperature dependent and it increased with decrease of the reaction temperature to $-30^{\circ} \mathrm{C}$ (Table 3, entries 11, 12). Once again addition of $\mathrm{PhCO}_{2} \mathrm{H}$ $(2.5 \mathrm{~mol} \%)$ turned out to be necessary to complete the reaction within $24 \mathrm{~h}$ (Table 3 , entry 12 ).

The optimal reaction conditions (Table 3 , entry 12) were applied to the 2-[bis(3,5-bistrifluoromethylphenyl)trimethylsilylanyloxymethyl]pyrrolidine-catalyzed reaction of different aliphatic aldehydes $\mathbf{2} \mathbf{h}-\mathbf{l}$ with compound $\mathbf{1}$ (Table 4 ). In all the cases, the reaction sequence involving Michael addi-

Table 4. 2-[Bis(3,5-bistrifluoromethylphenyl)trimethylsilylanyloxymethyl]pyrrolidine-catalyzed reaction of aliphatic aldehydes $\mathbf{2 h}-\mathbf{l}$ with ethyl 4-diethoxyphosphoryl-3-oxobutanoate (1). ${ }^{[a]}$

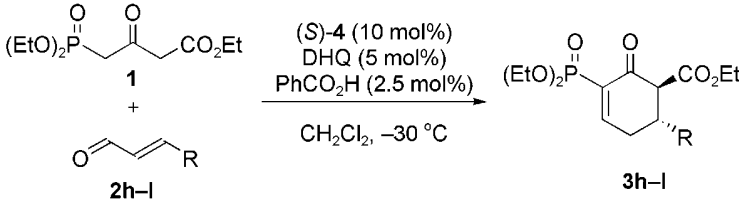

\begin{tabular}{lllll}
\hline & $\mathrm{R}$ & Yield [\%] & $e e[\%]^{[\mathrm{b}]}$ & $\mathrm{dr}^{[\mathrm{c}]}$ \\
\hline 1 & Et (2h) & 76 & 96 & $4: 1$ \\
2 & $i \operatorname{Pr}(\mathbf{2 i})^{[\mathrm{d}]}$ & 83 & 96 & $3: 1$ \\
3 & $n-\mathrm{C}_{7} \mathrm{H}_{15}(\mathbf{2} \mathbf{j})$ & 80 & 96 & $3: 1$ \\
4 & $(Z)-n$-hex-3-enyl (2 k) & 86 & 96 & $4: 1$ \\
5 & $\mathrm{CH}_{2} \mathrm{OBn}(\mathbf{2 l})$ & 72 & 94 & $4: 1$ \\
\hline
\end{tabular}

[a] All reactions were performed at $0.25 \mathrm{mmol}$ scale with two equivalents of the aldehyde in $\mathrm{CH}_{2} \mathrm{Cl}_{2}(1.25 \mathrm{~mL})$ for $24 \mathrm{~h}$. [b] Determined by HPLC on a chiral stationary phase. [c] Determined by ${ }^{31} \mathrm{P}$ NMR spectroscopy. [d] Performed using two equivalents of aldehyde $\mathbf{2 i}, 10 \mathrm{~mol} \%(S)-\mathbf{4}$ $5 \mathrm{~mol} \%$ DHQ, RT, $24 \mathrm{~h}$ 
tion and subsequent Knoevenagel condensation proceeded smoothly affording the products $\mathbf{3 h}-\mathbf{l}$ in good yields (72$86 \%$ ) and with excellent enantioselectivities (94-96\% ee). However, the diastereoselectivities were lower than those observed in the reactions with cinnamaldehyde (2a) and its derivatives $\mathbf{2 b}-\mathbf{g}$. The reaction conditions developed for the reaction of the aliphatic $\alpha, \beta$-unsaturated aldehydes in Table 3 are indicative of an interesting combination of covalent and non-covalent organocatalysis.

Mechanistic considerations: The proposed mechanism for the highly enantioselective, organocatalytic Michael-Knoevenagel domino reaction is outlined in Scheme 2. The Michael addition proceeds through the standard catalytic cycle reported in the literature for various similar iminium-catalyzed transformations. In the first step $\alpha, \beta$-unsaturated aldehyde $\mathbf{2}$ is activated by the formation of iminium ion $\mathbf{5}$ in the reversible reaction with amine catalyst 4 . Chemo- and regioselective nucleophilic attack by the C-2 methylene atom of $\mathbf{1}$ at the $\beta$-carbon of iminium ion $\mathbf{5}$ takes place preferentially from the less hindered $R e$-face of $\mathbf{5}$, to avoid unfavorable steric interactions with the bulky substituent at C-2 in the catalyst. Subsequent hydrolysis of the intermediate $\mathbf{6}$ releases the catalyst and leads to the formation of 7. Finally, compound 7 undergoes intramolecular Knoevenagel condensation, probably catalyzed by an amine in the case of aliphatic $\alpha, \beta$-unsaturated aldehydes, to afford $\mathbf{3}$. The absolute stereo-

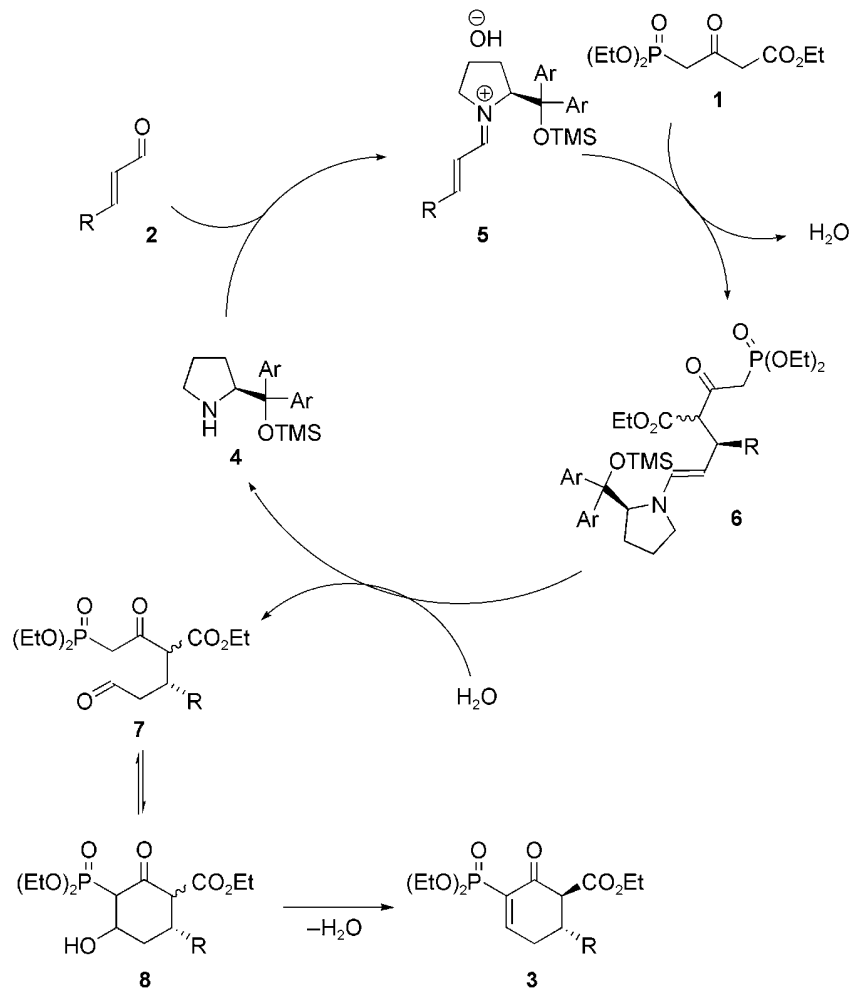

Scheme 2. Proposed mechanism for highly enantio- and diastereoselective organocatalytic Michael-Knoevenagel domino reaction leading to the formation of optically active 6-substituted-3-diethoxyphosphoryl-2oxocyclohex-3-enecarboxylates $\mathbf{3}$. chemistry of the C-6 stereogenic center in $\mathbf{3}$, controlled by the chirality of the catalyst, was unambiguously confirmed by single-crystal X-ray analysis of the compound 12a (Figure 1$)^{[10]}$ and is in accordance with the results obtained

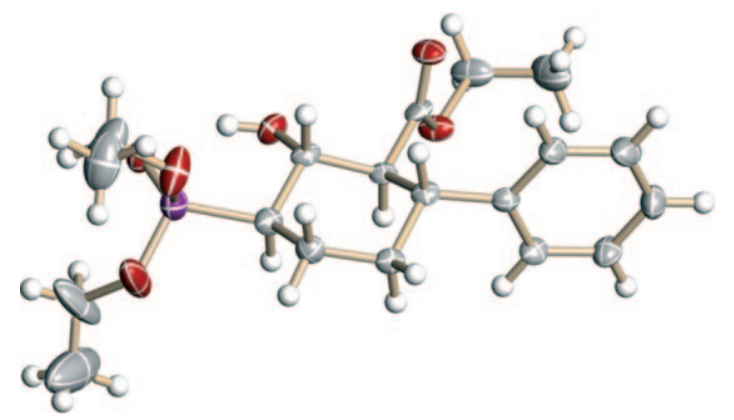

Figure 1. X-Ray structure of compound $\mathbf{1 2 a}$. C gray, $\mathrm{H}$ white, O red, P purple.

in other Michael additions catalyzed by 4 . The relative configuration of C-1 and C-6 stereogenic centers was based on NMR spectroscopic studies. The observed values of the coupling constants $\left({ }^{3} J(\mathrm{H} 1, \mathrm{H} 6)=10.7-13.2 \mathrm{~Hz}\right)$ clearly proved trans-diaxial arrangement of the protons at C- 1 and C-6 in the major diastereoisomers and, by analogy, allowed us to assign $1 S, 6 R$ absolute configuration to the products $\mathbf{3 a}-\mathbf{h}$ and $\mathbf{3} \mathbf{j}-\mathbf{l}$ and $1 S, 6 S$ to $\mathbf{3 i}$. Taking into account the mechanistic pathway, it is reasonable to assume that the absolute configuration at the C-6 stereogenic center in the minor diasteroisomers is the same as in the major ones. Both isomers differ in absolute configuration at $\mathrm{C}-1$. Values of the coupling constant $\left({ }^{3} \mathrm{~J}(\mathrm{H} 1, \mathrm{H} 6)=4.5-4.9 \mathrm{~Hz}\right)$ for the minor diastereoisomer indicates a cis-arrangement of the carboxylate moiety and $\mathrm{R}$ group. Formation of the minor diastereoisomer can be rationalized by the high acidity of the hydrogen at $\mathrm{C}-1$ making it prone to rapid epimerization.

Product elaborations: Optically active 6-substituted-3-diethoxyphosphoryl-2-oxocyclohex-3-enecarboxylates (3) were used for the preparation of various enantiomerically enriched cyclohexene and cyclohexane derivatives. Initially we attempted a hydrolysis-decarboxylation reaction as an entry to 5-substituted-2-(diethoxyphosphoryl)cyclohex-2-enones (11; Scheme 3). Since acid-catalyzed hydrolysis-decarboxylation of tert-butyl 2-oxocyclohex-3-enecarboxylates constitute a well-established strategy to access 2-cyclohexenones, we decided to perform the Michael-Knoevenagel domino reaction of tert-butyl 4-diethoxyphosphoryl-3-oxobutanoate (9) and cinnamaldehyde (2a) catalyzed by $\mathbf{4}$ to afford tert-butyl 2-oxocyclohex-3-enecarboxylate (10; Scheme 3). Methanesulfonic acid catalyzed hydrolysis-decarboxylation of $\mathbf{1 0}$ conducted in toluene at elevated temperature $\left(90^{\circ} \mathrm{C}\right)$ for $3 \mathrm{~h}$ yielded the target compound 2-diethoxyphosphoryl-5-phenylcyclohex-2-enone (11) in 96\% ee. More interestingly, compound $\mathbf{1 1}$ could easily be obtained in a one-pot reaction, by using toluene as a solvent for both addition-cyclization 


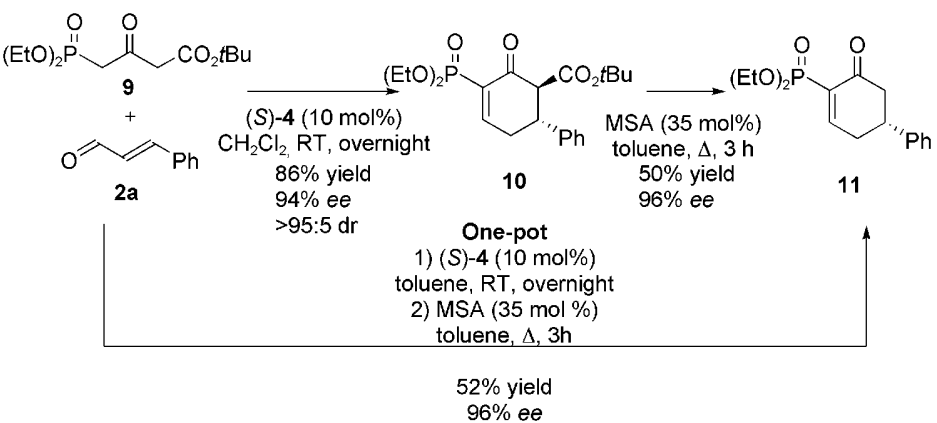

Scheme 3. Stepwise and one-pot approach for the synthesis of optically active 2-diethoxyphosphoryl-5-phenylcyclohex-2-enone $\mathbf{1 1}$.

and hydrolysis-decarboxylation steps, in good yield, and proceeded without lowering the optical purity achieved in the Michael addition step.

The synthetic potential of carboxylates $\mathbf{3}$ was further demonstrated in other stereoselective transformations (Scheme 4). To begin with, application of various reduction

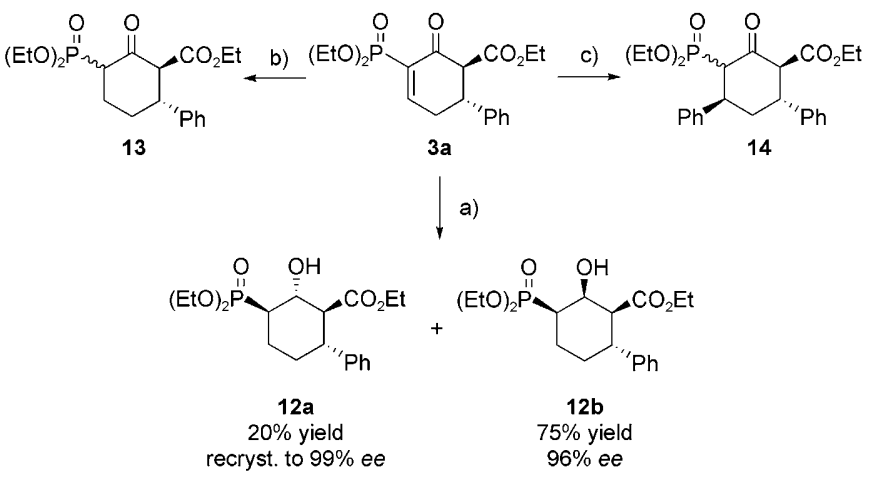

Scheme 4. Stereoselective transformations of $\mathbf{3 a}$. Reagents and conditions: a) $\mathrm{NaBH}_{4}$ (2 equiv), $\mathrm{CaCl}_{2}$ (1 equiv), $\mathrm{MeOH}, 0^{\circ} \mathrm{C}, 2 \mathrm{~h}$; b) $\mathrm{H}_{2}, 10 \%$ $\mathrm{Pd} / \mathrm{C}, \mathrm{MeOH}, \mathrm{RT}$, overnight; c) $\mathrm{PhMgBr}$ (1.2 equiv), CuI (1.2 equiv), $\mathrm{THF}, 0^{\circ} \mathrm{C}, 2 \mathrm{~h}$

conditions allowed us to obtain different phosphonates $\mathbf{1 2}$ and $\mathbf{1 3}$ in a highly chemo- and diastereoselective manner. For instance, reduction of $\mathbf{3 a}$ with $\mathrm{NaBH}_{4}$ in $\mathrm{MeOH}$ in the presence of $\mathrm{CaCl}_{2}$ afforded fully reduced $\beta$-hydroxyphosphonate 12 with four stereocenters in high yield and with good diastereoselectivity (4:1 dr). On the other hand, Pd/C-catalyzed hydrogenation of the double bond in $\mathbf{3 a}$ yielded $\beta$-ketophosphonate $\mathbf{1 3}$ as the only product. Moreover, compound 3a turned out to be an effective Michael acceptor. Conjugate addition of phenylmagnesium bromide to $\mathbf{3 a}$ in the presence of $\mathrm{CuI}$ afforded phosphonate 14. $\beta$-Ketophosphonates $\mathbf{1 3}$ and $\mathbf{1 4}$ were obtained as mixtures of two diastereoisomers differing in configuration at the C-3 stereogenic center and were not purified, but used as a crude for further elaborations. The results show that, due to the presence of the activated diethoxyphosphoryl moiety, compounds $\mathbf{1 3}$ and 14 could be efficiently utilized in Horner-WadsworthEmmons olefination of formaldehyde (Scheme 5).

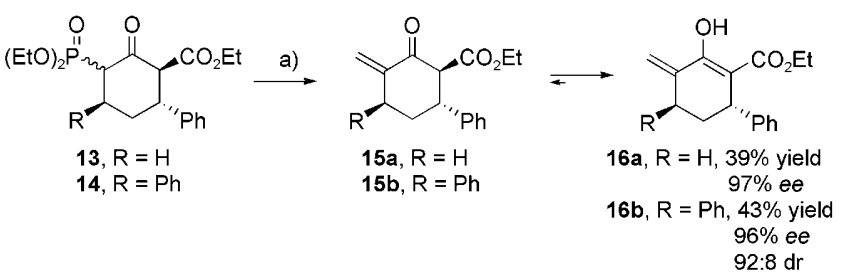

Scheme 5. Horner-Wadsworth-Emmons reaction of $\mathbf{1 3}$ and 14. Reagents and conditions: a) $\mathrm{K}_{2} \mathrm{CO}_{3}$ (3 equiv), $\mathrm{HCHO}$ (12 equiv), $\mathrm{THF} / \mathrm{H}_{2} \mathrm{O}, 0^{\circ} \mathrm{C}$, overnight.

This reaction led to the formation of particularly interesting $\alpha$-methyleneketones $\mathbf{1 5} \mathbf{a}$ and $\mathbf{b}$, existing as fully enolized forms $\mathbf{1 6} \mathbf{a}$ and $\mathbf{b}$, in a highly enantio- and diastereoselective manner and good overall yield. Assignment of relative and absolute configuration to the products 10-16 was based on single-crystal X-ray analysis of $\mathbf{1 2 a}$ and NMR spectroscopic studies.

\section{Conclusion}

In summary, we have developed a novel, stereoselective, organocatalytic Michael-Knoevenagel domino reaction of ethyl 4-diethoxyphosphoryl-3-oxobutanoate with $\alpha, \beta$-unsaturated aldehydes, catalyzed by diarylprolinol ether leading to enantiomerically enriched 6-substituted-3-diethoxyphosphoryl-2-oxocyclohex-3-enecarboxylates. Our methodology proved to be general, since a wide range of $\alpha, \beta$-unsaturated aldehydes with both aromatic and aliphatic substituents easily could be reacted, affording the corresponding products with high levels of stereocontrol. Furthermore, the application of optically active 6-substituted-3-diethoxyphosphoryl-2-oxocyclohex-3-enecarboxylates in various stereoselective transformations has been demonstrated providing access to a range of cyclohexene and cyclohexane derivatives with up to four stereocenters, thus indicating the high synthetic utility of the compounds obtained.

\section{Experimental Section}

General methods: NMR spectra were run at 400, 162, and $100 \mathrm{MHz}$ for ${ }^{1} \mathrm{H},{ }^{31} \mathrm{P}$, and ${ }^{13} \mathrm{C}$, respectively. Chemical shifts $(\delta)$ are reported in ppm relative to residual solvent signals $\left(\mathrm{CHCl}_{3}, \delta=7.26 \mathrm{ppm}\right.$ for ${ }^{1} \mathrm{H} \mathrm{NMR}$, $\mathrm{CDCl}_{3}, \delta=77.0 \mathrm{ppm}$ for ${ }^{13} \mathrm{C}$ NMR). The following abbreviations are used to indicate the multiplicity in ${ }^{1} \mathrm{H}$ NMR spectra: s, singlet; $d$, doublet; $t$, triplet; $\mathrm{m}$, multiplet; br, broad signal. ${ }^{13} \mathrm{C}$ NMR spectra were acquired on a broad-band decoupled mode. Chemical shifts for phosphorus are reported in ppm relative to phosphoric acid $\left(\mathrm{H}_{3} \mathrm{PO}_{4}, 0 \mathrm{ppm}\right)$ used as an external standard. All phosphorus nuclear magnetic resonance spectra are proton-decoupled. Mass spectra were recorded using electrospray $(\mathrm{ES}+)$ ionization techniques. Analytical TLC was performed by using precoated aluminum-backed plates and visualized by ultraviolet irradiation or $\mathrm{KMnO}_{4}$ dip. Melting points are uncorrected. Optical rotations were measured on a Perkin-Elmer 241 polarimeter. The enantiomeric excess (ee) of the products was determined by chiral stationary phase HPLC (Daicel Chiralpak AS/AD or Daicel Chiralcel OD/OJ columns).

Materials: Analytical grade solvents and commercially available reagents were used without further purification. Catalysts $(S)-\mathbf{4}$ and $(R)-\mathbf{4}$ were 
purchased from Sigma-Aldrich. Ethyl ${ }^{[11 a]}$ and tert-butyl ${ }^{[11 b]}$ 4-diethoxyphosphoryl-3-oxobutanoates $\mathbf{1}$ and $\mathbf{9}$ were prepared according to literature procedures. Flash chromatography (FC) was carried out by using Iatrobeads 6RS-8060 (spherical silica gel) or silica gel purchased from Fluka (silica gel 60, 230-400 mesh). Racemic samples were prepared by using a racemic mixture of the catalyst 4.

\section{General procedure for the preparation of alkyl 3-diethoxyphosphoryl-2-} oxocyclohex-3-enecarboxylates 3a-g and 10: An ordinary vial equipped with a magnetic stirring bar was charged with catalyst $4(0.025 \mathrm{mmol}$, $14.9 \mathrm{mg}), \mathrm{PhCO}_{2} \mathrm{H}(0.025 \mathrm{mmol}, 3 \mathrm{mg})$, and $\mathrm{CH}_{2} \mathrm{Cl}_{2}(1.25 \mathrm{~mL})$. Then, the solution was cooled to $-30^{\circ} \mathrm{C}$ and the $\alpha, \beta$-unsaturated aldehyde $\mathbf{2} \mathbf{a}-\mathbf{g}$ $(0.50 \mathrm{mmol})$ and 4-diethoxyphosphoryl-3-oxobutanoate $\mathbf{1}$ or $\mathbf{9}$ $(0.25 \mathrm{mmol})$ were added. The stirring was maintained at $-30^{\circ} \mathrm{C}$ for about $24 \mathrm{~h}$ until completion of the reaction (monitored by ${ }^{31} \mathrm{P}$ NMR spectroscopy) and then directly subjected to FC on Iatrobeads to afford the products $\mathbf{3} \mathbf{a}-\mathbf{g}$ or $\mathbf{1 0}$

(1S,6R)-Ethyl 3-(diethoxyphosphoryl)-2-oxo-6-phenylcyclohex-3-enecarboxylate (3a): Following the general procedure with catalyst $(S)-4$, compound 3 a was isolated by $\mathrm{FC}$ over Iatrobeads $\left(\mathrm{CH}_{2} \mathrm{Cl}_{2} / \mathrm{Et}_{2} \mathrm{O} 4: 1\right)$, as a yellow oil in $76 \%$ yield as single diastereoisomer $(\mathrm{dr}>95: 5) .{ }^{1} \mathrm{H}$ NMR $\left(\mathrm{CDCl}_{3}\right): \delta=7.93(\mathrm{ddd}, J=2.0,5.6,20.0 \mathrm{~Hz}, 1 \mathrm{H}), 7.33-7.20(\mathrm{~m}, 5 \mathrm{H})$, $4.25-4.08(\mathrm{~m}, 4 \mathrm{H}), 4.00(\mathrm{dq}, J=1.6,7.2 \mathrm{~Hz}, 2 \mathrm{H}), 3.71(\mathrm{~d}, J=12.9 \mathrm{~Hz}$, $1 \mathrm{H}), 3.65-3.55(\mathrm{~m}, 1 \mathrm{H}), 2.92-2.82(\mathrm{~m}, 1 \mathrm{H}), 2.75-2.64(\mathrm{~m}, 1 \mathrm{H}), 1.37-1.29$ $(\mathrm{m}, 6 \mathrm{H}), 1.01 \mathrm{ppm}(\mathrm{t}, J=7.2 \mathrm{~Hz}, 3 \mathrm{H}) ;{ }^{13} \mathrm{C} \mathrm{NMR}\left(\mathrm{CDCl}_{3}\right): \delta=191.2(\mathrm{~d}$, $\left.J_{\mathrm{CP}}=7.0 \mathrm{~Hz}\right), 168.5\left(\mathrm{~d}, J_{\mathrm{CP}}=1.3 \mathrm{~Hz}\right), 162.2\left(\mathrm{~d}, J_{\mathrm{CP}}=5.8 \mathrm{~Hz}\right), 140.3,131.2$ $\left(\mathrm{d}, J_{\mathrm{CP}}=183.2 \mathrm{~Hz}\right), 129.1(2 \times), 127.9,127.4(2 \times), 62.9\left(\mathrm{~d}, J_{\mathrm{CP}}=5.9 \mathrm{~Hz}\right.$, $2 \times), 61.2,60.5\left(\mathrm{~d}, J_{\mathrm{CP}}=7.6 \mathrm{~Hz}\right), 43.3,34.7\left(\mathrm{~d}, J_{\mathrm{CP}}=15.1 \mathrm{~Hz}\right), 16.6\left(\mathrm{~d}, J_{\mathrm{CP}}=\right.$ $6.3 \mathrm{~Hz}, 2 \times), 14.1 \mathrm{ppm} ;{ }^{31} \mathrm{P}$ NMR $\left(\mathrm{CDCl}_{3}\right): \delta=13.01 \mathrm{ppm}$; HRMS: $\mathrm{m} / z$ calcd for $\left[\mathrm{C}_{19} \mathrm{H}_{25} \mathrm{O}_{6} \mathrm{Na}\right]^{+}$: 403.1286; found: 403.1292; the ee was determined by HPLC analysis using a Chiralpak AD column (hexane/iPrOH $70: 30)$ : flow rate $1.0 \mathrm{~mL} \mathrm{~min}^{-1} ; \tau_{\text {major }}=9.5 \mathrm{~min}, \tau_{\text {minor }}=16.9 \mathrm{~min}(98 \% e e)$; $[\alpha]_{\mathrm{D}}^{\mathrm{RT}}=-7.24\left(c=0.72\right.$ in $\left.\mathrm{CHCl}_{3}\right)$.

(1R,6S)-Ethyl 3-(diethoxyphosphoryl)-2-oxo-6-phenylcyclohex-3-enecarboxylate (ent-3): Following the general procedure with catalyst $(R)-4$, compound ent-3a was isolated by $\mathrm{FC}$ using Iatrobeads $\left(\mathrm{CH}_{2} \mathrm{Cl}_{2} / \mathrm{Et}_{2} \mathrm{O}\right.$ $4: 1)$, as a yellow oil in $76 \%$ yield as single diastereoisomer $(\mathrm{dr}>95: 5)$. The $e e$ was determined by HPLC analysis using a Chiralpak AD column (hexane $/ \mathrm{PrOH}$ 70:30): flow rate $1.0 \mathrm{~mL} \mathrm{~min}^{-1} ; \tau_{\text {major }}=16.7 \mathrm{~min}, \tau_{\text {minor }}=$ $9.8 \min (98 \% e e) ;[\alpha]_{\mathrm{D}}^{\mathrm{RT}}=+7.63\left(c=1.42\right.$ in $\left.\mathrm{CHCl}_{3}\right)$. Spectral data were identical to compound $\mathbf{3 a}$.

(1S,6R)-Ethyl 3-(diethoxyphosphoryl)-6-(4-nitrophenyl)-2-oxocyclohex3-enecarboxylate (3b): Following the general procedure with catalyst $(S)$ 4, $3 \mathbf{b}$ was isolated by $\mathrm{FC}$ using Iatrobeads $\left(\mathrm{CH}_{2} \mathrm{Cl}_{2} / \mathrm{Et}_{2} \mathrm{O} 4: 1\right)$, as a yellow oil in $95 \%$ yield as a mixture of diastereoisomers (87:13). Major diastereoisomer: ${ }^{1} \mathrm{H}$ NMR $\left(\mathrm{CDCl}_{3}\right): \delta=8.14(\mathrm{~d}, J=8.7 \mathrm{~Hz}, 2 \mathrm{H}), 7.87(\mathrm{ddd}, J=$ 2.2, 5.7, $20.0 \mathrm{~Hz}, 1 \mathrm{H}), 7.38(\mathrm{~d}, J=8.7 \mathrm{~Hz}, 2 \mathrm{H}), 4.16-4.08(\mathrm{~m}, 4 \mathrm{H}), 4.03$ $3.91(\mathrm{~m}, 2 \mathrm{H}), 3.83-3.76(\mathrm{~m}, 1 \mathrm{H}), 3.74(\mathrm{~d}, J=12.8 \mathrm{~Hz}, 1 \mathrm{H}), 2.86-2.80(\mathrm{~m}$, $1 \mathrm{H}), 2.70-2.62(\mathrm{~m}, 1 \mathrm{H}), 1.30-1.26(\mathrm{~m}, 6 \mathrm{H}), 1.02 \mathrm{ppm}(\mathrm{t}, J=7.1 \mathrm{~Hz}, 3 \mathrm{H})$; ${ }^{13} \mathrm{C} \mathrm{NMR}\left(\mathrm{CDCl}_{3}\right): \delta=189.9\left(\mathrm{~d}, J_{\mathrm{CP}}=7.0 \mathrm{~Hz}\right), 167.7\left(\mathrm{~d}, J_{\mathrm{CP}}=1.3 \mathrm{~Hz}\right)$, $160.7\left(\mathrm{~d}, J_{\mathrm{CP}}=5.8 \mathrm{~Hz}\right), 150.7,147.4,131.3\left(\mathrm{~d}, J_{\mathrm{CP}}=183.5 \mathrm{~Hz}\right), 128.2(2 \times)$, $124.1(2 \times), 62.8\left(\mathrm{~d}, J_{\mathrm{CP}}=5.5 \mathrm{~Hz}, 2 \times\right), 61.4,59.6\left(\mathrm{~d}, J_{\mathrm{CP}}=5.7 \mathrm{~Hz}\right), 42.8$, $33.9\left(\mathrm{~d}, J_{\mathrm{CP}}=15.2 \mathrm{~Hz}\right), 16.3\left(\mathrm{~d}, J_{\mathrm{CP}}=6.1 \mathrm{~Hz}, 2 \times\right), 13.9 \mathrm{ppm}$; minor diastereoisomer (representative signals): ${ }^{1} \mathrm{H} \mathrm{NMR}\left(\mathrm{CDCl}_{3}\right): \delta=8.05(\mathrm{~d}, J=$ $8.7 \mathrm{~Hz}, 2 \mathrm{H}), 7.29$ (d, $J=8.7 \mathrm{~Hz}, 2 \mathrm{H}), 7.09$ (ddd, $J=2.3,6.1,19.3 \mathrm{~Hz}, 1 \mathrm{H}$ ). ${ }^{13} \mathrm{C} \mathrm{NMR}\left(\mathrm{CDCl}_{3}\right): \delta=160.7\left(\mathrm{~d}, J_{\mathrm{CP}}=5.0 \mathrm{~Hz}\right), 147.3,127.8(2 \times), 123.6$ $(2 \times), 62.5\left(\mathrm{~d}, J_{\mathrm{CP}}=5.0 \mathrm{~Hz}, 2 \times\right), 60.8\left(\mathrm{~d}, J_{\mathrm{CP}}=8.9 \mathrm{~Hz}\right), 32.5\left(\mathrm{~d}, J_{\mathrm{CP}}=\right.$ $15.7 \mathrm{~Hz}), 16.2\left(\mathrm{~d}, J_{\mathrm{CP}}=10.6 \mathrm{~Hz}, 2 \times\right), 13.7 \mathrm{ppm} ;{ }^{31} \mathrm{P} \mathrm{NMR}\left(\mathrm{CDCl}_{3}\right): \delta=$ $13.65(13 \%), 12.40 \mathrm{ppm}(87 \%)$; HRMS : $\mathrm{m} / z$ calcd for $\left[\mathrm{C}_{19} \mathrm{H}_{24} \mathrm{NO}_{8} \mathrm{PNa}\right]^{+}$: 448.1137; found: 448.1139; the ee was determined by HPLC analysis using a Chiralpak $\mathrm{AD}$ column (hexane/iPrOH 70:30): flow rate $0.8 \mathrm{~mL} \mathrm{~min}^{-1} ; \tau_{\text {major }}=23.4 \mathrm{~min}, \tau_{\text {minor }}=42.9 \mathrm{~min}(98 \% e e) ;[\alpha]_{\mathrm{D}}^{\mathrm{RT}}=-15.57$ $\left(c=1.32\right.$ in $\left.\mathrm{CHCl}_{3}\right)$.

(1S,6R)-Ethyl 3-(diethoxyphosphoryl)-2-oxo-6-(4-(trifluoromethyl)phenyl)cyclohex-3-enecarboxylate $\mathbf{( 3 c ) : ~ F o l l o w i n g ~ t h e ~ g e n e r a l ~ p r o c e d u r e ~}$ with catalyst $(S)-\mathbf{4}$, compound $\mathbf{3 c}$ was isolated by FC using Iatrobeads $\left(\mathrm{CH}_{2} \mathrm{Cl}_{2} / \mathrm{Et}_{2} \mathrm{O} 4: 1\right)$, as a yellow oil in $81 \%$ yield as single diastereoisomer $(\mathrm{dr}>95: 5) .{ }^{1} \mathrm{H}$ NMR $\left(\mathrm{CDCl}_{3}\right): \delta=7.92(\mathrm{ddd}, J=2.0,5.6,20.0 \mathrm{~Hz}, 1 \mathrm{H})$, $7.58(\mathrm{~d}, J=8.0 \mathrm{~Hz}, 2 \mathrm{H}), 7.35(\mathrm{~d}, J=8.0 \mathrm{~Hz}, 2 \mathrm{H}), 4.25-4.09(\mathrm{~m}, 4 \mathrm{H}), 4.09-$ $3.98(\mathrm{~m}, 2 \mathrm{H}), 3.79-3.74(\mathrm{~m}, 2 \mathrm{H}), 2.91-2.82(\mathrm{~m}, 1 \mathrm{H}), 2.73-2.63(\mathrm{~m}, 1 \mathrm{H})$ $1.35-1.30(\mathrm{~m}, 6 \mathrm{H}), 1.04 \mathrm{ppm}(\mathrm{t}, J=6.8 \mathrm{~Hz}, 3 \mathrm{H}) ;{ }^{13} \mathrm{C} \mathrm{NMR}\left(\mathrm{CDCl}_{3}\right): \delta=$ $190.5\left(\mathrm{~d}, J_{\mathrm{CP}}=7.4 \mathrm{~Hz}\right), 168.2,161.4\left(\mathrm{~d}, J_{\mathrm{CP}}=5.9 \mathrm{~Hz}\right), 144.4,131.4\left(\mathrm{~d}, J_{\mathrm{CP}=}\right.$ $183.4 \mathrm{~Hz}), 130.2\left(\mathrm{q}, J_{\mathrm{CF}=} 32.4 \mathrm{~Hz}\right), 127.8(2 \times), 126.1\left(\mathrm{q}, J_{\mathrm{CF}=} 3.6 \mathrm{~Hz}, 2 \times\right)$ $124.1\left(\mathrm{~d}, J_{\mathrm{CF}=} 270.7 \mathrm{~Hz}\right), 63.0\left(\mathrm{dd}, J_{\mathrm{CP}}=2.2,5.9 \mathrm{~Hz}, 2 \times\right), 61.5,60.1\left(\mathrm{~d}, J_{\mathrm{CP}=}\right.$ $7.7 \mathrm{~Hz}), 43.1,34.4\left(\mathrm{~d}, J_{\mathrm{CP}=} 15.2 \mathrm{~Hz}\right), 16.5\left(\mathrm{~d} . J_{\mathrm{CP}=} 5.9 \mathrm{~Hz}, 2 \times\right), 14.0 \mathrm{ppm}$; ${ }^{31} \mathrm{P}$ NMR $\quad\left(\mathrm{CDCl}_{3}\right): \quad \delta=12.63 \mathrm{ppm}$; HRMS: $\mathrm{m} / \mathrm{z}$ calcd for $\left[\mathrm{C}_{20} \mathrm{H}_{24} \mathrm{~F}_{3} \mathrm{O}_{6} \mathrm{PNa}\right]^{+}$: 471.1160; found: 471.1164; the ee was determined by HPLC analysis using a Chiralcel OJ column (hexane/ $i \mathrm{PrOH} 90: 10$ ): flow rate $1.0 \mathrm{~mL} \mathrm{~min}^{-1} ; \tau_{\text {major }}=36.6 \mathrm{~min}, \tau_{\text {minor }}=16.3 \mathrm{~min}(97 \% e e) ;[\alpha]_{\mathrm{D}}^{\mathrm{RT}}=$ $-11.4\left(c=1.09\right.$ in $\left.\mathrm{CHCl}_{3}\right)$.

(1S,6R)-Ethyl 3-(diethoxyphosphoryl)-6-(2-methoxyphenyl)-2-oxocyclohex-3-enecarboxylate (3d): Following the general procedure with catalyst $(S)-4$, compound 3d was isolated by $\mathrm{FC}$ using Iatrobeads $\left(\mathrm{CH}_{2} \mathrm{Cl}_{2} / \mathrm{Et}_{2} \mathrm{O}\right.$ $4: 1)$, as a yellow oil in $94 \%$ yield as a mixture of diastereoisomers $(92: 8)$. Major diastereoisomer: ${ }^{1} \mathrm{H} \mathrm{NMR}\left(\mathrm{CDCl}_{3}\right): \delta=7.90$ (ddd, $J=2.6,5.5$, $20.2 \mathrm{~Hz}, 1 \mathrm{H}), 7.17(\mathrm{dt}, J=1.6,7.9 \mathrm{~Hz}, 1 \mathrm{H}), 7.04(\mathrm{dt}, J=1.5,7.9 \mathrm{~Hz}, 1 \mathrm{H})$, 6.84-6.80 (m, 2 H), 4.18-4.06 (m, 4 H), $4.06(\mathrm{~d}, J=13.2 \mathrm{~Hz}, 1 \mathrm{H}), 3.95(\mathrm{dq}$ $J=0.6,7.1 \mathrm{~Hz}, 2 \mathrm{H}), 3.90-3.80(\mathrm{~m}, 1 \mathrm{H}), 3.78(\mathrm{~s}, 3 \mathrm{H}), 2.85$ (dddd, $J=2.6$, $4.5,10.1,20.1 \mathrm{~Hz}, 1 \mathrm{H}), 2.75(\mathrm{ddt}, J=2.1,5.4,20.2 \mathrm{~Hz}, 1 \mathrm{H}), 1.30-1.25(\mathrm{~m}$, $6 \mathrm{H}), 0.96 \mathrm{ppm}(\mathrm{t}, J=7.1 \mathrm{~Hz}, 3 \mathrm{H}) ;{ }^{13} \mathrm{C} \mathrm{NMR}\left(\mathrm{CDCl}_{3}\right): \delta=192.0\left(\mathrm{~d}, J_{\mathrm{CP}}=\right.$ $6.8 \mathrm{~Hz}), 168.9\left(\mathrm{~d}, J_{\mathrm{CP}}=1.3 \mathrm{~Hz}\right), 163.4\left(\mathrm{~d}, J_{\mathrm{CP}}=5.8 \mathrm{~Hz}\right), 157.8,130.7(\mathrm{~d}$, $\left.J_{\mathrm{CP}}=182.8 \mathrm{~Hz}\right), 129.0,128.9,128.0,120.9,111.3,62.9\left(\mathrm{~d}, J_{\mathrm{CP}}=4.7 \mathrm{~Hz}, 2 \times\right)$, $61.1,58.6\left(\mathrm{~d}, J_{\mathrm{CP}}=7.7 \mathrm{~Hz}\right), 55.6,39.8,32.6\left(\mathrm{~d}, J_{\mathrm{CP}}=14.9 \mathrm{~Hz}\right), 16.6\left(\mathrm{~d}, J_{\mathrm{CP}}=\right.$ $6.2 \mathrm{~Hz}, 2 \times), 14.1 \mathrm{ppm}$; minor diastereoisomer (representative signals): ${ }^{1} \mathrm{H}$ NMR $\left(\mathrm{CDCl}_{3}\right): \delta=8.11(\mathrm{ddd}, J=2.0,6.3,20.5 \mathrm{~Hz}, 1 \mathrm{H}), 7.09(\mathrm{dd}, J=$ $1.3,7.7 \mathrm{~Hz}, 1 \mathrm{H}), 0.87 \mathrm{ppm}(\mathrm{t}, J=7.1 \mathrm{~Hz}, 3 \mathrm{H}) ;{ }^{13} \mathrm{C} \mathrm{NMR}\left(\mathrm{CDCl}_{3}\right): \delta=$ $165.5\left(\mathrm{~d}, J_{\mathrm{CP}}=6.1 \mathrm{~Hz}\right), 128.7,127.1,120.6,110.5,60.7,57.0\left(\mathrm{~d}, J_{\mathrm{CP}}=\right.$ $7.6 \mathrm{~Hz}), 55.5,35.7,28.3 \mathrm{ppm}\left(\mathrm{d}, J_{\mathrm{CP}}=14.4 \mathrm{~Hz}\right) ;{ }^{31} \mathrm{P} \mathrm{NMR}\left(\mathrm{CDCl}_{3}\right): \delta=$ $14.26(8 \%), 13.52 \mathrm{ppm}(92 \%)$; HRMS: $\mathrm{m} / \mathrm{z}$ calcd for $\left[\mathrm{C}_{20} \mathrm{H}_{27} \mathrm{O}_{7} \mathrm{PNa}\right]^{+}$ 433.1392; found: 433.1396; the ee was determined by HPLC analysis using a Chiralpak AD column (hexane $/ \mathrm{PrOH}$ 70:30): flow rate $1.0 \mathrm{~mL} \mathrm{~min}^{-1} ; \tau_{\text {major }}=10.6 \mathrm{~min}, \tau_{\text {minor }}=20.6 \mathrm{~min}(97 \% e e) ;[\alpha]_{\mathrm{D}}^{\mathrm{RT}}=-12.89$ $\left(c=1.03\right.$ in $\left.\mathrm{CHCl}_{3}\right)$.

(1S,6R)-Ethyl 3-(diethoxyphosphoryl)-6-(3-methoxyphenyl)-2-oxocyclohex-3-enecarboxylate (3e): Following the general procedure with catalyst $(S)-4$, compound $3 \mathbf{e}$ was isolated by $\mathrm{FC}$ using Iatrobeads $\left(\mathrm{CH}_{2} \mathrm{Cl}_{2} / \mathrm{Et}_{2} \mathrm{O}\right.$ $4: 1$ ), as a yellow oil in $76 \%$ yield as single diastereoisomer $(\mathrm{dr}>95: 5)$. ${ }^{1} \mathrm{H}$ NMR $\left(\mathrm{CDCl}_{3}\right): \delta=7.94(\mathrm{ddd}, J=2.3,5.7,20.1 \mathrm{~Hz}, 1 \mathrm{H}), 7.23(\mathrm{~d}, J=$ $7.9 \mathrm{~Hz}, 1 \mathrm{H}), 6.82-6.75(\mathrm{~m}, 3 \mathrm{H}), 4.24-4.12(\mathrm{~m}, 4 \mathrm{H}), 4.04(\mathrm{dq}, J=1.8$, $7.1 \mathrm{~Hz}, 2 \mathrm{H}), 3.78(\mathrm{~s}, 3 \mathrm{H}), 3.76(\mathrm{~d}, J=12.8 \mathrm{~Hz}, 1 \mathrm{H}), 3.71-3.64(\mathrm{~m}, 1 \mathrm{H})$, 2.91-2.84 (m, 1 H), 2.74-2.65 (m, 1 H), 1.39 (dt, $J=0.5,7.1 \mathrm{~Hz}, 3 \mathrm{H}), 1.33$ $(\mathrm{dt}, J=0.5,7.1 \mathrm{~Hz}, 3 \mathrm{H}), 1.06 \mathrm{ppm}(\mathrm{t}, J=7.1 \mathrm{~Hz}, 3 \mathrm{H}) ;{ }^{13} \mathrm{C} \mathrm{NMR}\left(\mathrm{CDCl}_{3}\right)$ : $\delta=190.9\left(\mathrm{~d}, J_{\mathrm{CP}}=6.5 \mathrm{~Hz}\right), 168.2\left(\mathrm{~d}, J_{\mathrm{CP}}=1.1 \mathrm{~Hz}\right), 161.9\left(\mathrm{~d}, J_{\mathrm{CP}}=5.8 \mathrm{~Hz}\right)$, $159.7,141.7,130.9\left(\mathrm{~d}, J_{\mathrm{CP}}=182.2 \mathrm{~Hz}\right), 129.8,119.2,113.1,112.6,62.7(\mathrm{~d}$ $\left.J_{\mathrm{CP}}=6.0 \mathrm{~Hz}, 2 \times\right), 61.0,60.2\left(\mathrm{~d}, J_{\mathrm{CP}}=7.7 \mathrm{~Hz}\right), 55.1,43.1,34.5\left(\mathrm{~d}, J_{\mathrm{CP}}=\right.$ $15.1 \mathrm{~Hz}), 16.3\left(\mathrm{~d}, J_{\mathrm{CP}}=6.2 \mathrm{~Hz}, 2 \times\right), 13.8 \mathrm{ppm} ;{ }^{31} \mathrm{P} \mathrm{NMR}\left(\mathrm{CDCl}_{3}\right): \delta=$ $13.0 \mathrm{ppm}$; HRMS: $\mathrm{m} / \mathrm{z}$ calcd for $\left[\mathrm{C}_{20} \mathrm{H}_{27} \mathrm{O}_{7} \mathrm{PNa}\right]^{+}: 433.1392$; found 433.1392.; the ee was determined by HPLC analysis using a Chiralpak AD column (hexane $/ \mathrm{PrOH} 70: 30$ ): flow rate $1.0 \mathrm{~mL} \mathrm{~min}^{-1} ; \tau_{\text {major }}=$ $11.7 \mathrm{~min}, \tau_{\text {minor }}=17.4 \mathrm{~min}(97 \% e e) ;[\alpha]_{\mathrm{D}}^{\mathrm{RT}}=-8.9\left(c=1.47\right.$ in $\left.\mathrm{CHCl}_{3}\right)$.

(1S,6R)-Ethyl 6-(biphenyl-4-yl)-3-(diethoxyphosphoryl)-2-oxocyclohex-3enecarboxylate (3f): Following the general procedure with catalyst $(S)-4$, compound $\mathbf{3 f}$ was isolated by $\mathrm{FC}$ using Iatrobeads $\left(\mathrm{CH}_{2} \mathrm{Cl}_{2} / \mathrm{Et}_{2} \mathrm{O} 4: 1\right)$, as a yellow oil in $78 \%$ yield as single diastereoisomer $(\mathrm{dr}>95: 5) .{ }^{1} \mathrm{H}$ NMR $\left(\mathrm{CDCl}_{3}\right): \delta=7.89(\mathrm{ddd}, \mathrm{J}=2.0,5.6,20.0 \mathrm{~Hz}, 1 \mathrm{H}), 7.48(\mathrm{dd}, J=1.6,7.6 \mathrm{~Hz}$, $4 \mathrm{H}), 7.35(\mathrm{t}, J=7.6 \mathrm{~Hz}, 2 \mathrm{H}), 7.28-7.17(\mathrm{~m}, 3 \mathrm{H}), 4.20-4.04(\mathrm{~m}, 4 \mathrm{H}), 3.97$ $(\mathrm{dq}, J=1.2,7.2 \mathrm{~Hz}, 2 \mathrm{H}), 3.74(\mathrm{~d}, J=12.8 \mathrm{~Hz}, 1 \mathrm{H}) 3.72-3.63(\mathrm{~m}, 1 \mathrm{H})$, 2.87-2.79 (m, 1H), 2.71-2.62 (m, $1 \mathrm{H}), 1.27(\mathrm{dt}, J=5.6,7.2 \mathrm{~Hz}, 6 \mathrm{H})$, $0.98 \mathrm{ppm}(\mathrm{t}, J=7.2 \mathrm{~Hz}, 3 \mathrm{H}) ;{ }^{13} \mathrm{CNMR}\left(\mathrm{CDCl}_{3}\right): \delta=191.1 \quad\left(\mathrm{~d}, J_{\mathrm{CP}}=\right.$ $6.9 \mathrm{~Hz}), 168.5,162.1\left(\mathrm{~d}, J_{\mathrm{CP}}=5.9 \mathrm{~Hz}\right), 140.7,140.6,139.3,131.2\left(\mathrm{~d}, J_{\mathrm{CP}}=\right.$ $183.3 \mathrm{~Hz}), 129.0(2 \times), 127.7(2 \times), 127.7(2 \times), 127.6,127.1(2 \times), 63.0(\mathrm{~d}$, $\left.J_{\mathrm{CP}}=5.4 \mathrm{~Hz}, 2 \times\right), 61.3,60.6\left(\mathrm{~d}, J_{\mathrm{CP}}=7.7 \mathrm{~Hz}\right), 43.0,34.7\left(\mathrm{~d}, J_{\mathrm{CP}}=15.1 \mathrm{~Hz}\right)$, $16.5\left(\mathrm{~d}, J_{\mathrm{CP}}=6.0 \mathrm{~Hz}, 2 \times\right), 14.1 \mathrm{ppm} ;{ }^{31} \mathrm{P} \mathrm{NMR}\left(\mathrm{CDCl}_{3}\right): \delta=13.04 \mathrm{ppm}$; HRMS: $m / z$ calcd for $\left[\mathrm{C}_{25} \mathrm{H}_{29} \mathrm{O}_{6} \mathrm{PNa}\right]^{+}: 479.1599$; found: 479.1595 ; the $e e$ was determined by HPLC analysis using a Chiralcel OD column (hexane $/ \mathrm{PrOH} 70: 30$ ): flow rate $0.8 \mathrm{~mL} \mathrm{~min}^{-1} ; \tau_{\text {major }}=30.9 \mathrm{~min}, \tau_{\text {minor }}=$ $24.5 \min (97 \% e e) ;[\alpha]_{\mathrm{D}}^{\mathrm{RT}}=-18.80\left(c=1.65\right.$ in $\left.\mathrm{CHCl}_{3}\right)$. 
(1S,6R)-Ethyl 3-(diethoxyphosphoryl)-6-(furan-2-yl)-2-oxocyclohex-3enecarboxylate (3g): Following the general procedure with catalyst $(S)-\mathbf{4}$, compound $\mathbf{3 g}$ was isolated by $\mathrm{FC}$ using Iatrobeads $\left(\mathrm{CH}_{2} \mathrm{Cl}_{2} / \mathrm{Et}_{2} \mathrm{O} 4: 1\right)$, as a yellow oil in $71 \%$ yield as a mixture of diastereoisomers (9:1). Major diastereoisomer: ${ }^{1} \mathrm{H}$ NMR $\left(\mathrm{CDCl}_{3}\right): \delta=7.90(\mathrm{ddd}, J=3.2,5.2,20.4 \mathrm{~Hz}$, $1 \mathrm{H}), 7.30(\mathrm{dd}, J=0.8,2.0 \mathrm{~Hz}, 1 \mathrm{H}), 6.25(\mathrm{dd}, J=2.0,3.2 \mathrm{~Hz}, 1 \mathrm{H}), 6.07$ (d, $J=3.2 \mathrm{~Hz}, 1 \mathrm{H}), 4.23-4.05(\mathrm{~m}, 6 \mathrm{H}), 3.89-3.83(\mathrm{~m}, 1 \mathrm{H}), 3.71(\mathrm{~d}, J=$ $10.8 \mathrm{~Hz}, 1 \mathrm{H}), 2.97-2.76(\mathrm{~m}, 2 \mathrm{H}), 1.34-1.25(\mathrm{~m}, 6 \mathrm{H}), 1.17 \mathrm{ppm}(\mathrm{t}, J=$ $7.2 \mathrm{~Hz}, 3 \mathrm{H} ;{ }^{13} \mathrm{C}$ NMR $\left(\mathrm{CDCl}_{3}\right): \delta=190.3\left(\mathrm{~d}, J_{\mathrm{CP}}=7.2 \mathrm{~Hz}\right), 168.5 .161 .8(\mathrm{~d}$, $\left.J_{\mathrm{CP}}=5.7 \mathrm{~Hz}\right), 153.4,142.3,131.1\left(\mathrm{~d}, J_{\mathrm{CP}}=182.1 \mathrm{~Hz}\right), 110.5,106.7,62.9(\mathrm{~d}$, $\left.J_{\mathrm{CP}}=5.8 \mathrm{~Hz}, 2 \times\right), 61.7,58.4\left(J_{\mathrm{CP}}=7.5 \mathrm{~Hz}\right), 36.6,31.1\left(\mathrm{~d}, J_{\mathrm{CP}}=15.0 \mathrm{~Hz}\right)$, $16.5\left(\mathrm{~d}, J_{\mathrm{CP}}=6.3 \mathrm{~Hz}, 2 \times\right), 14.2 \mathrm{ppm}$; minor diastereoisomer (representative signals): ${ }^{1} \mathrm{H}$ NMR $\left(\mathrm{CDCl}_{3}\right): \delta=8.07$ (ddd, $\left.J=1.6,5.2,20.4 \mathrm{~Hz}, 1 \mathrm{H}\right)$, $7.33(\mathrm{~d}, J=1.6 \mathrm{~Hz}, 1 \mathrm{H}), 6.30(\mathrm{dd}, J=1.6,3.2 \mathrm{~Hz}, 1 \mathrm{H}), 6.12(\mathrm{~d}, J=3.2 \mathrm{~Hz}$, $1 \mathrm{H}), 3.24-3.10(\mathrm{~m}, 2 \mathrm{H}), 1.07 \mathrm{ppm}(\mathrm{t}, J=7.2 \mathrm{~Hz}, 3 \mathrm{H}) ;{ }^{31} \mathrm{P} \mathrm{NMR}\left(\mathrm{CDCl}_{3}\right)$ : $\delta=13.54(10.0 \%), \quad 13.96 \mathrm{ppm} \quad(90.0 \%) ; \quad$ HRMS: $\mathrm{m} / \mathrm{z}$ calcd for $\left[\mathrm{C}_{17} \mathrm{H}_{23} \mathrm{O}_{7} \mathrm{PNa}\right]^{+}$: 393.1079; found: 393.1050 ; the ee was determined by HPLC analysis using a Chiralpak AD column (hexane/ $i \mathrm{PrOH}$ 60:40): flow rate $0.7 \mathrm{~mL} \mathrm{~min}^{-1} ; \tau_{\text {major }}=9.0 \mathrm{~min}, \tau_{\text {minor }}=11.0 \min (97 \% e e) ;[\alpha]_{\mathrm{D}}^{\mathrm{RT}}=$ $-10.83\left(c=1.21\right.$ in $\left.\mathrm{CHCl}_{3}\right)$.

(1S,6R)-tert-Butyl 3-(diethoxyphosphoryl)-2-oxo-6-phenylcyclohex-3enecarboxylate (10): Following the general procedure with catalyst $(S)-4$ in the absence of $\mathrm{PhCO}_{2} \mathrm{H}$ at RT, compound $\mathbf{1 0}$ was isolated by FC using Iatrobeads $\left(\mathrm{CH}_{2} \mathrm{Cl}_{2} / \mathrm{Et}_{2} \mathrm{O} 4: 1\right)$, as a yellow oil in $87 \%$ yield as single diastereoisomer $(\mathrm{dr}>95: 5) .{ }^{1} \mathrm{H}$ NMR $\left(\mathrm{CDCl}_{3}\right): \delta=7.89$ (ddd, $J=2.4$, 5.6, $20.4 \mathrm{~Hz}, 1 \mathrm{H}), 7.31-7.10(\mathrm{~m}, 5 \mathrm{H}), 4.23-4.04(\mathrm{~m}, 4 \mathrm{H}), 3.64-3.55(\mathrm{~m}, 2 \mathrm{H})$, 2.87-2.77 (m, 1H), 2.71-2.59 (m, 1H), $1.30(\mathrm{dq}, J=1.6,6.8 \mathrm{~Hz}, 6 \mathrm{H})$, $1.18 \mathrm{ppm}(\mathrm{s}, 9 \mathrm{H}) ;{ }^{13} \mathrm{C}$ NMR $\left(\mathrm{CDCl}_{3}\right): \delta=191.4\left(\mathrm{~d}, J_{\mathrm{CP}}=6.7 \mathrm{~Hz}\right), 167.4$, $161.9\left(\mathrm{dd}, J_{\mathrm{CP}=1.6}, 5.8 \mathrm{~Hz}\right), 140.4,131.1\left(\mathrm{~d}, J_{\mathrm{CP}=} 182.8 \mathrm{~Hz}\right), 128.8(2 \times)$, 127.6, $127.4(2 \times), 81.8,62.8\left(\mathrm{t}, J_{\mathrm{CP}}=6.0 \mathrm{~Hz}, 2 \times\right), 61.3\left(\mathrm{~d}, J_{\mathrm{CP}=} 7.5 \mathrm{~Hz}\right)$, 43.6, $34.9\left(\mathrm{~d}, J_{\mathrm{CP}=1} 15.2 \mathrm{~Hz}\right), 27.7(\mathrm{~s}, 3 \times), 16.5 \mathrm{ppm}\left(\mathrm{d}, J_{\mathrm{CP}=} 6.3 \mathrm{~Hz}, 2 \times\right)$; ${ }^{31} \mathrm{P}$ NMR $\left(\mathrm{CDCl}_{3}\right): \delta=13.40 \mathrm{ppm}$; HRMS : $m / z$ calcd for $\left[\mathrm{C}_{21} \mathrm{H}_{29} \mathrm{O}_{6} \mathrm{PNa}\right]^{+}$: 431.1599; found: 431.1604; the ee was determined by HPLC analysis using a Chiralpak AD column (hexane $/ \mathrm{PrOH}$ 80:20): flow rate $1.0 \mathrm{~mL} \mathrm{~min}^{-1} ; \tau_{\text {major }}=20.8 \mathrm{~min}, \tau_{\text {minor }}=30.8 \mathrm{~min} \quad(94 \% e e) ;[\alpha]_{\mathrm{D}}^{\mathrm{RT}}=-4.7$ $\left(c=0.92\right.$ in $\left.\mathrm{CHCl}_{3}\right)$.

General procedure for the preparation of ethyl 3-diethoxyphosphoryl-2oxocyclohex-3-enecarboxylates 3h-l: An ordinary vial equipped with a magnetic stirring bar was charged with catalyst $4(0.025 \mathrm{mmol}, 14.9 \mathrm{mg})$, dihydroquinine (DHQ; $0.0125 \mathrm{mmol}, 4.1 \mathrm{mg}), \mathrm{PhCO}_{2} \mathrm{H}(0.00625 \mathrm{mmol}$, $0.8 \mathrm{mg}$ ), and $\mathrm{CH}_{2} \mathrm{Cl}_{2}(1.25 \mathrm{~mL})$. The resulting solution was cooled to $-30^{\circ} \mathrm{C}$ and the $\alpha, \beta$-unsaturated aldehyde $2(0.50 \mathrm{mmol})$ and compound 1 $(0.25 \mathrm{mmol}, 66.6 \mathrm{mg})$ were added. The stirring was maintained at $-30^{\circ} \mathrm{C}$ for about $24 \mathrm{~h}$ until completion of the reaction (monitored by ${ }^{31} \mathrm{P}$ NMR spectroscopy) and then directly subjected to FC on Iatrobeads to afford the products $3 \mathbf{h}-\mathbf{l}$.

(1S,6R)-Ethyl 3-(diethoxyphosphoryl)-6-ethyl-2-oxocyclohex-3-enecarboxylate (3h): Following the general procedure with catalyst $(S)-\mathbf{4}$, compound $3 \mathbf{h}$ was isolated by $\mathrm{FC}$ using Iatrobeads $\left(\mathrm{CH}_{2} \mathrm{Cl}_{2} / \mathrm{Et}_{2} \mathrm{O} 4: 1\right)$, as a yellow oil in $76 \%$ yield as a mixture of diastereoisomers (4:1). Major diastereoisomer: ${ }^{1} \mathrm{H}$ NMR $\left(\mathrm{CDCl}_{3}\right): \delta=7.84(\mathrm{ddd}, J=2.9,5.2,20.1 \mathrm{~Hz}, 1 \mathrm{H})$, 4.19-4.02 (m, 9H), 3.19 (d, $J=10.8 \mathrm{~Hz}, 1 \mathrm{H}), 2.69$ (ddt, $J=2.6,5.1$, $20.0 \mathrm{~Hz}, 1 \mathrm{H}), 2.53-2.37(\mathrm{~m}, 1 \mathrm{H}), 2.25-2.16(\mathrm{~m}, 1 \mathrm{H}), 1.49-1.40(\mathrm{~m}, 2 \mathrm{H})$, $1.27-1.20(\mathrm{~m}, 9 \mathrm{H}), 0.88 \mathrm{ppm}(\mathrm{t}, J=7.5 \mathrm{~Hz}, 3 \mathrm{H}) ;{ }^{13} \mathrm{C} \mathrm{NMR}\left(\mathrm{CDCl}_{3}\right): \delta=$ $191.4\left(\mathrm{~d}, J_{\mathrm{CP}}=6.6 \mathrm{~Hz}\right), 169.21,162.5\left(\mathrm{~d}, J_{\mathrm{CP}}=5.9 \mathrm{~Hz}\right), 130.5\left(\mathrm{~d}, J_{\mathrm{CP}}=\right.$ $183.2 \mathrm{~Hz}), 62.6\left(\mathrm{~d}, J_{\mathrm{CP}}=6.4 \mathrm{~Hz}\right), 62.5\left(\mathrm{~d}, J_{\mathrm{CP}}=7.0 \mathrm{~Hz}\right), 61.2,59.8\left(\mathrm{~d}, J_{\mathrm{CP}}=\right.$ $7.6 \mathrm{~Hz}), 37.8,30.5\left(\mathrm{~d}, J_{\mathrm{CP}}=15.0 \mathrm{~Hz}\right), 26.2,16.3\left(\mathrm{~d}, J_{\mathrm{CP}}=6.4 \mathrm{~Hz}\right), 16.2(\mathrm{~d}$, $\left.J_{\mathrm{CP}}=6.3 \mathrm{~Hz}\right), 14.0,10.4 \mathrm{ppm}$; minor diastereoisomer (representative signals): ${ }^{1} \mathrm{H}$ NMR $\left(\mathrm{CDCl}_{3}\right): \delta=7.93(\mathrm{ddd}, J=2.9,4.8,20.2 \mathrm{~Hz}, 1 \mathrm{H}), 3.46$ $(\mathrm{dd}, J=2.2,4.9 \mathrm{~Hz}, 1 \mathrm{H}), 0.93 \mathrm{ppm}(\mathrm{t}, J=7.4 \mathrm{~Hz}, 3 \mathrm{H}) ;{ }^{13} \mathrm{C} \mathrm{NMR}\left(\mathrm{CDCl}_{3}\right)$ : $\delta=167.6,164.7\left(\mathrm{~d}, J_{\mathrm{CP}}=5.6 \mathrm{~Hz}\right), 61.1,36.7,25.6,13.9,10.3 \mathrm{ppm} ;{ }^{31} \mathrm{P} \mathrm{NMR}$ $\left(\mathrm{CDCl}_{3}\right): \delta=14.14(20 \%), 13.38 \mathrm{ppm}(80 \%)$; HRMS: $\mathrm{m} / \mathrm{z}$ calcd for $\left[\mathrm{C}_{15} \mathrm{H}_{25} \mathrm{O}_{6} \mathrm{PNa}\right]^{+}:$355.1286; found: 355.1291 ; the ee was determined by HPLC analysis using a Chiralpak AD column (hexane $/ i \operatorname{PrOH} 80: 20$ ): flow rate $1.0 \mathrm{~mL} \mathrm{~min}^{-1} ; \tau_{\text {major }}=9.8 \mathrm{~min}, \tau_{\text {minor }}=10.8 \min (96 \% e e) ;[\alpha]_{\mathrm{D}}^{\mathrm{RT}}=$ $-14.2\left(c=1.19\right.$ in $\left.\mathrm{CHCl}_{3}\right)$.

(1S,6S)-Ethyl 3-(diethoxyphosphoryl)-6-isopropyl-2-oxocyclohex-3-enecarboxylate (3i): Following the general procedure with catalyst $(S)-\mathbf{4}$, in the absence of $\mathrm{PhCO}_{2} \mathrm{H}$ at RT, compound $\mathbf{3 i}$ was isolated by FC using Iatrobeads $\left(\mathrm{CH}_{2} \mathrm{Cl}_{2} / \mathrm{Et}_{2} \mathrm{O} 4: 1\right)$, as a yellow oil in $83 \%$ yield as a mixture of diastereoisomers (3:1). Major diastereoisomer: ${ }^{1} \mathrm{H} \mathrm{NMR}\left(\mathrm{CDCl}_{3}\right): \delta=$ 7.87 (ddd, $J=2.8,5.4,20.1 \mathrm{~Hz}, 1 \mathrm{H}), 4.17(\mathrm{q}, J=7.1 \mathrm{~Hz}, 2 \mathrm{H}), 4.13-4.01$ $(\mathrm{m}, 4 \mathrm{H}), 3.32(\mathrm{~d}, J=11.4 \mathrm{~Hz}, 1 \mathrm{H}), 2.58-2.49(\mathrm{~m}, 1 \mathrm{H}), 2.46-2.39(\mathrm{~m}, 1 \mathrm{H})$, $2.32-2.23(\mathrm{~m}, 1 \mathrm{H}), 1.72-1.60(\mathrm{~m}, 1 \mathrm{H}), 1.27-1.20(\mathrm{~m}, 9 \mathrm{H}), 0.91(\mathrm{~d}, J=$ $6.9 \mathrm{~Hz}, 3 \mathrm{H}), 0.82 \mathrm{ppm}(\mathrm{d}, J=6.9 \mathrm{~Hz}, 3 \mathrm{H}) ;{ }^{13} \mathrm{C}$ NMR $\left(\mathrm{CDCl}_{3}\right): \delta=191.8$ $\left(\mathrm{d}, J_{\mathrm{CP}}=6.8 \mathrm{~Hz}\right), 169.3,163.1\left(\mathrm{~d}, J_{\mathrm{CP}}=5.5 \mathrm{~Hz}\right), 130.6\left(\mathrm{~d}, J_{\mathrm{CP}}=183.9 \mathrm{~Hz}\right)$, $62.6\left(\mathrm{~d}, J_{\mathrm{CP}}=5.8 \mathrm{~Hz}\right), 62.5\left(\mathrm{~d}, J_{\mathrm{CP}}=5.8 \mathrm{~Hz}\right), 61.2,58.5\left(\mathrm{~d}, J_{\mathrm{CP}}=7.6 \mathrm{~Hz}\right)$, $42.1,28.9,26.0\left(\mathrm{~d}, J_{\mathrm{CP}}=15.0 \mathrm{~Hz}\right), 20.3,16.5,16.3\left(\mathrm{~d}, J_{\mathrm{CP}}=6.2 \mathrm{~Hz}\right), 16.2(\mathrm{~d}$, $J_{\mathrm{CP}}=6.4 \mathrm{~Hz}$ ), $14.1 \mathrm{ppm}$; minor diastereoisomer (representative signals): ${ }^{1} \mathrm{H}$ NMR $\left(\mathrm{CDCl}_{3}\right): \delta=7.95(\mathrm{ddd}, J=2.1,5.8,20.4 \mathrm{~Hz}, 1 \mathrm{H}), 3.60(\mathrm{dd}, J=$ $2.3,4.5 \mathrm{~Hz}, 1 \mathrm{H}), 2.65-2.58(\mathrm{~m}, 1 \mathrm{H}), 1.85-1.73(\mathrm{~m}, 1 \mathrm{H}), 1.17(\mathrm{t}, J=$ $7.1 \mathrm{~Hz}, 3 \mathrm{H}), 0.95(\mathrm{~d}, J=6.5 \mathrm{~Hz}, 3 \mathrm{H}), 0.91 \mathrm{ppm}(\mathrm{d}, J=6.6 \mathrm{~Hz}, 3 \mathrm{H})$; ${ }^{13} \mathrm{C}$ NMR $\left(\mathrm{CDCl}_{3}\right): \delta=191.5\left(\mathrm{~d}, J_{\mathrm{CP}}=6.6 \mathrm{~Hz}\right), 165.2\left(\mathrm{~d}, J_{\mathrm{CP}}=6.0 \mathrm{~Hz}\right), 62.3$ $\left(\mathrm{d}, J_{\mathrm{CP}}=5.0 \mathrm{~Hz}, 2 \times\right), 43.9,30.1,29.2\left(\mathrm{~d}, J_{\mathrm{CP}}=14.7 \mathrm{~Hz}\right), 20.4,20.4$, $14.0 \mathrm{ppm} ;{ }^{31} \mathrm{P} \mathrm{NMR} \quad\left(\mathrm{CDCl}_{3}\right): \quad \delta=14.12 \quad(25 \%), 13.33 \mathrm{ppm} \quad(75 \%)$; HRMS: $\mathrm{m} / z$ calcd for $\left[\mathrm{C}_{16} \mathrm{H}_{27} \mathrm{O}_{6} \mathrm{PNa}\right]^{+}: 369.1443$; found: 369.1448 ; the $e e$ was determined by HPLC analysis using a Chiralpak AD column (hexane $/ i \mathrm{PrOH} 80: 20$ ): flow rate $1.0 \mathrm{~mL} \mathrm{~min}^{-1} ; \tau_{\text {major }}=8.9 \mathrm{~min}, \tau_{\text {minor }}=$ $10.1 \min (96 \% e e) ;[\alpha]_{\mathrm{D}}^{\mathrm{RT}}=-16.12\left(c=1.67\right.$ in $\left.\mathrm{CHCl}_{3}\right)$.

(1S,6R)-Ethyl 3-(diethoxyphosphoryl)-6-heptyl-2-oxocyclohex-3-enecarboxylate (3j): Following the general procedure with catalyst $(S)-\mathbf{4}$, compound $3 \mathbf{j}$ was isolated by $\mathrm{FC}$ using Iatrobeads $\left(\mathrm{CH}_{2} \mathrm{Cl}_{2} / \mathrm{Et}_{2} \mathrm{O} 4: 1\right)$, as a yellow oil in $80 \%$ yield as a mixture of diastereoisomers $(3: 1)$. Major diastereoisomer: ${ }^{1} \mathrm{H}$ NMR $\left(\mathrm{CDCl}_{3}\right): \delta=7.88(\mathrm{ddd}, J=2.9,5.1,20.2 \mathrm{~Hz}, 1 \mathrm{H})$, 4.25-4.07 (m, 6H), $3.23(\mathrm{~d}, J=10.7 \mathrm{~Hz}, 1 \mathrm{H}), 2.76(\mathrm{ddt}, J=2.5,5.0$, $20.0 \mathrm{~Hz}, 1 \mathrm{H}), 2.56-2.48(\mathrm{~m}, 1 \mathrm{H}), 2.30-2.21(\mathrm{~m}, 1 \mathrm{H}), 1.35-1.21(\mathrm{~m}, 21 \mathrm{H})$, $0.87 \mathrm{ppm}(\mathrm{t}, J=6.7 \mathrm{~Hz}, 3 \mathrm{H}) ;{ }^{13} \mathrm{C} \mathrm{NMR}\left(\mathrm{CDCl}_{3}\right): \delta=191.7\left(\mathrm{~d}, J_{\mathrm{CP}}=\right.$ $6.9 \mathrm{~Hz}), 169.5,162.7\left(\mathrm{~d}, J_{\mathrm{CP}}=5.7 \mathrm{~Hz}\right), 130.8\left(\mathrm{~d}, J_{\mathrm{CP}}=183.2 \mathrm{~Hz}\right), 62.9(\mathrm{t}$, $\left.J_{\mathrm{CP}}=6.6 \mathrm{~Hz}, 2 \times\right), 61.5,60.4\left(\mathrm{~d}, J_{\mathrm{CP}}=7.5 \mathrm{~Hz}\right), 36.7,33.8,31.9,31.4(\mathrm{~d}$, $\left.J_{\mathrm{CP}}=15.0 \mathrm{~Hz}\right), 29.6,29.3,26.2,22.8,16.6\left(\mathrm{~d}, J_{\mathrm{CP}}=6.2 \mathrm{~Hz}\right), 16.6\left(\mathrm{~d}, J_{\mathrm{CP}}=\right.$ $6.4 \mathrm{~Hz}), 14.4,14.2 \mathrm{ppm}$; minor diastereoisomer (representative signals): ${ }^{1} \mathrm{H}$ NMR $\left(\mathrm{CDCl}_{3}\right): \delta=7.99(\mathrm{dt}, J=3.8,20.0 \mathrm{~Hz}, 1 \mathrm{H}), 3.49 \mathrm{ppm}(\mathrm{dd}, J=$ $2.2,4.8 \mathrm{~Hz}, 1 \mathrm{H}) ;{ }^{13} \mathrm{C} \mathrm{NMR}\left(\mathrm{CDCl}_{3}\right): \delta=167.9,164.9\left(\mathrm{~d}, J_{\mathrm{CP}}=5.7 \mathrm{~Hz}\right)$, $63.8\left(\mathrm{~d}, J_{\mathrm{CP}}=5.8 \mathrm{~Hz}\right), 62.6\left(\mathrm{~d}, J_{\mathrm{CP}}=5.1 \mathrm{~Hz}\right), 61.3,57.3\left(\mathrm{~d}, J_{\mathrm{CP}}=7.4 \mathrm{~Hz}\right)$, $37.2,32.9,31.9,31.1\left(\mathrm{~d}, J_{\mathrm{CP}}=14.6 \mathrm{~Hz}\right), 26.7 \mathrm{ppm} ;{ }^{31} \mathrm{P} \mathrm{NMR}\left(\mathrm{CDCl}_{3}\right): \delta=$ $14.16(25 \%), 13.41 \mathrm{ppm}(75 \%)$; HRMS: $\mathrm{m} / z$ calcd for $\left[\mathrm{C}_{20} \mathrm{H}_{35} \mathrm{O}_{6} \mathrm{PNa}\right]^{+}$: 425.2069; found: 425.2071; the ee was determined by HPLC analysis using a Chiralpak AD column (hexane $/ i \operatorname{PrOH}$ 80:20): flow rate $1.0 \mathrm{~mL} \mathrm{~min}^{-1} ; \tau_{\text {major }}=8.4 \mathrm{~min}, \tau_{\text {minor }}=9.5 \mathrm{~min} \quad(96 \% e e) ; \quad[\alpha]_{\mathrm{D}}^{\mathrm{RT}}=-15.18$ $\left(c=1.59\right.$ in $\left.\mathrm{CHCl}_{3}\right)$.

(1S,6R)-Ethyl 3-(diethoxyphosphoryl)-6-((Z)-hex-3-enyl)-2-oxocyclohex3-enecarboxylate (3k): Following the general procedure with catalyst $(S)$ 4, compound $3 \mathbf{k}$ was isolated by $\mathrm{FC}$ using Iatrobeads $\left(\mathrm{CH}_{2} \mathrm{Cl}_{2} / \mathrm{Et}_{2} \mathrm{O} 4: 1\right)$, as a yellow oil in $86 \%$ yield as a mixture of diastereoisomers (4:1). Major diastereoisomer: ${ }^{1} \mathrm{H} \mathrm{NMR}\left(\mathrm{CDCl}_{3}\right)$ : $\delta=7.82$ (ddd, $J=3.0,5.1$, $20.2 \mathrm{~Hz}, 1 \mathrm{H}), 5.37-5.30(\mathrm{~m}, 1 \mathrm{H}), 5.23-5.16(\mathrm{~m}, 1 \mathrm{H}), 4.20-4.02(\mathrm{~m}, 6 \mathrm{H})$ $3.20(\mathrm{~d}, J=10.7 \mathrm{~Hz}, 1 \mathrm{H}), 2.72(\mathrm{dtd}, J=2.5,5.1,19.9 \mathrm{~Hz}, 1 \mathrm{H}), 2.54-2.43$ $(\mathrm{m}, 1 \mathrm{H}), 2.27-2.17(\mathrm{~m}, 1 \mathrm{H}), 2.10-1.92(\mathrm{~m}, 4 \mathrm{H}), 1.47-1.41(\mathrm{~m}, 1 \mathrm{H}), 1.36$ $1.30(\mathrm{~m}, 1 \mathrm{H}), 1.27-1.16(\mathrm{~m}, 9 \mathrm{H}), 0.89 \mathrm{ppm}(\mathrm{t}, J=7.5 \mathrm{~Hz}, 3 \mathrm{H}) ;{ }^{13} \mathrm{C}$ NMR $\left(\mathrm{CDCl}_{3}\right): \delta=191.5\left(\mathrm{~d}, J_{\mathrm{CP}}=6.8 \mathrm{~Hz}\right), 169.4,162.7\left(\mathrm{~d}, J_{\mathrm{CP}}=5.7 \mathrm{~Hz}\right), 133.0$ $130.9\left(\mathrm{~d}, J_{\mathrm{CP}}=183.5 \mathrm{~Hz}\right), 127.5,62.9\left(\mathrm{~d}, J_{\mathrm{CP}}=6.4 \mathrm{~Hz}\right), 62.8\left(\mathrm{~d}, J_{\mathrm{CP}}=\right.$ $6.1 \mathrm{~Hz}), 61.5,60.3\left(\mathrm{~d}, J_{\mathrm{CP}}=7.5 \mathrm{~Hz}\right), 36.3,33.7,31.2\left(\mathrm{~d}, J_{\mathrm{CP}}=15.0 \mathrm{~Hz}\right), 29.9$, $23.8,20.7,16.6\left(\mathrm{~d}, J_{\mathrm{CP}}=6.1 \mathrm{~Hz}\right), 16.5\left(\mathrm{~d}, J_{\mathrm{CP}}=6.3 \mathrm{~Hz}\right), 14.3 \mathrm{ppm}$; minor diastereoisomer (representative signals): ${ }^{1} \mathrm{H}$ NMR $\left(\mathrm{CDCl}_{3}\right): \delta=7.92(\mathrm{dt}$ $J=4.0,20.3 \mathrm{~Hz}, 1 \mathrm{H}), 3.43 \mathrm{ppm}(\mathrm{dd}, J=2.2,4.9 \mathrm{~Hz}, 1 \mathrm{H}) ;{ }^{13} \mathrm{C} \mathrm{NMR}$ $\left(\mathrm{CDCl}_{3}\right): \delta=164.7\left(\mathrm{~d}, J_{\mathrm{CP}}=5.8 \mathrm{~Hz}\right), 133.1,127.5,61.4,57.9\left(\mathrm{~d}, J_{\mathrm{CP}}=\right.$ $7.6 \mathrm{~Hz}), 36.5,32.7,31.0\left(\mathrm{~d}, J_{\mathrm{CP}}=14.7 \mathrm{~Hz}\right), 25.5,24.4,14.4 \mathrm{ppm} ;{ }^{31} \mathrm{P}$ NMR $\left(\mathrm{CDCl}_{3}\right): \delta=14.07(20 \%), 13.33 \mathrm{ppm}(80 \%)$; HRMS: $\mathrm{m} / \mathrm{z}$ calcd for $\left[\mathrm{C}_{19} \mathrm{H}_{31} \mathrm{O}_{6} \mathrm{PNa}\right]^{+}:$409.1756; found: 409.1754; the $e e$ was determined by HPLC analysis using a Chiralpak AD column (hexane $/ i \operatorname{PrOH} 80: 20$ ): flow rate $1.0 \mathrm{~mL} \mathrm{~min}^{-1} ; \tau_{\text {major }}=10.6 \mathrm{~min}, \tau_{\text {minor }}=12.0 \mathrm{~min} \quad(96 \% \mathrm{ee})$; $[\alpha]_{\mathrm{D}}^{\mathrm{RT}}=-7.66\left(c=1.59\right.$ in $\left.\mathrm{CHCl}_{3}\right)$.

(1S,6R)-Ethyl 6-(benzyloxymethyl)-3-(diethoxyphosphoryl)-2-oxocyclohex-3-enecarboxylate (3I): Following the general procedure with catalyst $(S)-\mathbf{4}$, compound 31 was isolated by $\mathrm{FC}$ using Iatrobeads $\left(\mathrm{CH}_{2} \mathrm{Cl}_{2} / \mathrm{Et}_{2} \mathrm{O}\right.$ $4: 1$ ), as a yellow oil in $72 \%$ yield as a mixture of diastereoisomers $(4: 1)$. 
Major diastereoisomer: ${ }^{1} \mathrm{H} \mathrm{NMR}\left(\mathrm{CDCl}_{3}\right): \delta=7.88$ (ddd, $J=3.2,4.8$, $20.0 \mathrm{~Hz}, 1 \mathrm{H}), 7.36-7.24(\mathrm{~m}, 5 \mathrm{H}), 4.49-4.41(\mathrm{~m}, 2 \mathrm{H}), 4.23-4.02(\mathrm{~m}, 6 \mathrm{H})$, $3.52(\mathrm{~d}, J=11.2 \mathrm{~Hz}, 1 \mathrm{H}), 3.42(\mathrm{~d}, J=4.0 \mathrm{~Hz}, 2 \mathrm{H}), 2.83-2.71(\mathrm{~m}, 1 \mathrm{H})$, 2.70-2.57 (m, 2H), 1.33-1.22 ppm (m, 9H); ${ }^{13} \mathrm{C} \mathrm{NMR}\left(\mathrm{CDCl}_{3}\right): \delta=191.5$ $(\mathrm{d}, J=6.8 \mathrm{~Hz}), 169.2,162.9(\mathrm{dd}, J=2.2,5.9 \mathrm{~Hz}), 137.9,130.7(\mathrm{~d}, J=$ $182.2 \mathrm{~Hz}), 128.6(2 \times), 128.0,127.8(2 \times), 73.6,71.0,62.9(\mathrm{~d}, J=5.7 \mathrm{~Hz}$, $2 \times), 61.5,56.8(\mathrm{~d}, J=7.5 \mathrm{~Hz}), 37.5,29.3\left(\mathrm{~d}, J_{\mathrm{CP}}=15.1 \mathrm{~Hz}\right), 16.5(\mathrm{~d}, J=$ $6.2 \mathrm{~Hz}), 16.5\left(\mathrm{~d}, J_{\mathrm{CP}}=6.3 \mathrm{~Hz}\right), 14.3 \mathrm{ppm}$; minor diastereoisomer (representative signals): ${ }^{1} \mathrm{H} N \mathrm{NM}\left(\mathrm{CDCl}_{3}\right): \delta=8.01-7.93(\mathrm{~m}, 1 \mathrm{H}), 3.64$ $3.59 \mathrm{ppm}(\mathrm{m}, 1 \mathrm{H}) ;{ }^{13} \mathrm{C} \mathrm{NMR}\left(\mathrm{CDCl}_{3}\right): \delta=164.2 \mathrm{ppm}(\mathrm{dd}, J=2.5,6.4 \mathrm{~Hz})$; ${ }^{31} \mathrm{P}$ NMR $\left(\mathrm{CDCl}_{3}\right): \delta=13.82(20 \%), 13.31 \mathrm{ppm}(80 \%)$; HRMS: $\mathrm{m} / \mathrm{z}$ calcd for $\left[\mathrm{C}_{21} \mathrm{H}_{29} \mathrm{O}_{7} \mathrm{PNa}\right]^{+}$: 447.1549 ; found: 447.1543 ; the $e e$ was determined by HPLC analysis using a Chiralpak AD column (hexane/iPrOH $75: 25$ ): flow rate $1.0 \mathrm{~mL} \mathrm{~min}^{-1} ; \tau_{\text {major }}=9.7 \mathrm{~min}, \tau_{\text {minor }}=12.0 \mathrm{~min}(94 \% e e) ;[\alpha]_{\mathrm{D}}^{\mathrm{RT}}=$ $-32.30\left(c=1.71\right.$ in $\left.\mathrm{CHCl}_{3}\right)$.

\section{Preparation of diethyl 6-oxo-4-phenylcyclohex-1-enylphosphonate (11)}

Method A-decarboxylation of tert-butyl 3-diethoxyphosphoryl-2-oxocyclohex-3-enecarboxylate (10): Methanesulfonic acid $(50.0 \mathrm{~mol} \%)$ was added to a heated $\left(90^{\circ} \mathrm{C}\right)$ solution $(0.2 \mathrm{M})$ of phosphonate $\mathbf{1 0}$ in toluene $(1.25 \mathrm{~mL})$ and the resulting mixture was stirred at elevated temperature for $3 \mathrm{~h}$, cooled to RT, concentrated in vacuo, and directly subjected to FC on Iatrobeads to afford the product $\mathbf{1 1}$

Method B-one-pot synthesis: An ordinary vial equipped with a magnetic stirring bar was charged with catalyst $(S)-\mathbf{4}(0.025 \mathrm{mmol}, 14.9 \mathrm{mg})$ and toluene $(1.25 \mathrm{~mL})$. The $\alpha, \beta$-unsaturated aldehyde $\mathbf{2 a}(1.00 \mathrm{mmol})$ and tert-butyl 4-diethoxyphosphoryl-3-oxobutanoate $9(0.25 \mathrm{mmol})$ were added. The stirring was maintained at RT for about $20 \mathrm{~h}$ until completion of the domino Michael-Knoevenagel reaction (monitored by ${ }^{31} \mathrm{P}$ NMR spectroscopy); then the mixture was heated to $90^{\circ} \mathrm{C}$ and methanesulfonic acid was added $(0.125 \mathrm{mmol}, 50 \mathrm{~mol} \%)$. The reaction was stirred at elevated temperature for $3 \mathrm{~h}$, cooled to RT, concentrated in vacuo, and directly subjected to FC on Iatrobeads to afford the product $\mathbf{1 1}$.

(6R)-Diethyl 6-oxo-4-phenylcyclohex-1-enylphosphonate (11) Following one of the procedures above, compound $\mathbf{1 1}$ was isolated by FC using Iatrobeads $\left(\mathrm{CH}_{2} \mathrm{Cl}_{2} / \mathrm{Et}_{2} \mathrm{O} 4: 1\right)$, as a colorless oil in $50 \%$ or $52 \%$ yield respectively. ${ }^{1} \mathrm{H}$ NMR $\left(\mathrm{CDCl}_{3}\right): \delta=7.95(\mathrm{ddd}, J=2.2,5.6,20.4 \mathrm{~Hz}, 1 \mathrm{H})$, $7.34(\mathrm{~d}, J=7.6 \mathrm{~Hz}, 2 \mathrm{H}), 7.26(\mathrm{t}, J=7.3 \mathrm{~Hz}, 1 \mathrm{H}), 7.21(\mathrm{~d}, J=7.5 \mathrm{~Hz}, 2 \mathrm{H})$, 4.25-4.10 (m, 4H), 3.4-3.32 (m, 1H), 2.86-2.61 (m, 4H), $1.34(\mathrm{t}, J=$ $7.1 \mathrm{~Hz}, 3 \mathrm{H}), 1.33 \mathrm{ppm}(\mathrm{t}, J=7.1 \mathrm{~Hz}, 3 \mathrm{H}) ;{ }^{13} \mathrm{C} \mathrm{NMR}\left(\mathrm{CDCl}_{3}\right): \delta=195.6$ $\left(\mathrm{d}, J_{\mathrm{CP}}=6.1 \mathrm{~Hz}\right), 162.2\left(\mathrm{~d}, J_{\mathrm{CP}}=6.0 \mathrm{~Hz}\right), 142.2,131.6\left(\mathrm{~d}, J_{\mathrm{CP}}=183.5 \mathrm{~Hz}\right)$, $128.9(2 \times), 127.2,126.5(2 \times), 62.6\left(\mathrm{~d}, J_{\mathrm{CP}}=4.9 \mathrm{~Hz}\right), 62.5\left(\mathrm{~d}, J_{\mathrm{CP}}=5.6 \mathrm{~Hz}\right)$, $45.3\left(\mathrm{~d}, J_{\mathrm{CP}}=7.7 \mathrm{~Hz}\right), 40.2,34.6\left(\mathrm{~d}, J_{\mathrm{CP}}=15.2 \mathrm{~Hz}\right), 16.4\left(\mathrm{~d}, J_{\mathrm{CP}}=6.3 \mathrm{~Hz}\right)$, $16.4 \mathrm{ppm}\left(\mathrm{d}, J_{\mathrm{CP}}=6.3 \mathrm{~Hz}\right) ;{ }^{31} \mathrm{P}$ NMR $\left(\mathrm{CDCl}_{3}\right): \delta=14.30 \mathrm{ppm}$; HRMS: $\mathrm{m} / z$ calcd for $\left[\mathrm{C}_{16} \mathrm{H}_{21} \mathrm{O}_{4} \mathrm{Na}\right]^{+}$: 331.1075 ; found: 331.1076; the ee was determined by HPLC analysis using a Chiralpak AS column (hexane $/ i \mathrm{PrOH}$ 95:5): flow rate $1.0 \mathrm{mLmin}^{-1} ; \tau_{\text {major }}=30.0 \mathrm{~min}, \tau_{\text {minor }}=35.2 \mathrm{~min}(96 \% e e)$. $[\alpha]_{\mathrm{D}}^{\mathrm{RT}}=-8.75\left(c=1.00\right.$ in $\left.\mathrm{CHCl}_{3}\right)$.

Procedure for the preparation of ethyl 3-(diethoxyphosphoryl)-2-hydroxy-6-phenylcyclohexanecarboxylate (12): A solution of $\mathbf{3 a}$ (76 mg, $0.2 \mathrm{mmol})$ in $\mathrm{CH}_{2} \mathrm{Cl}_{2}(1 \mathrm{~mL})$ was added to a solution of $\mathrm{CaCl}_{2}$ in $\mathrm{MeOH}$ $(0.4 \mathrm{M}, 1.5 \mathrm{~mL})$. The mixture was left stirring for approximately $30 \mathrm{~min}$ at RT, after which it was cooled to $-30^{\circ} \mathrm{C}$ and $\mathrm{NaBH}_{4}$ was added $(0.30 \mathrm{mmol}, 11.4 \mathrm{mg})$. The reaction was left stirring for $45 \mathrm{~min}$ (followed by TLC), diluted with $\mathrm{Et}_{2} \mathrm{O}(2 \mathrm{~mL})$, and quenched with aqueous $1 \mathrm{~m}$ $0.6 \mathrm{~mL} \mathrm{NaHSO}_{3}$. The phases were separated and the aqueous phase extracted two times with $\mathrm{CH}_{2} \mathrm{Cl}_{2}(20 \mathrm{~mL})$, the organic phases were collected, dried over $\mathrm{MgSO}_{4}$ and concentrated in vacuo. The residue was purified by FC on Iatrobeads to afford the compound $\mathbf{1 2}$.

$(1 S, 2 R, 3 R, 6 R)$-Ethyl 3-(diethoxyphosphoryl)-2-hydroxy-6-phenylcyclohexanecarboxylate (12 a; minor diastereomer): Compound 12 a was isolated by FC using Iatrobeads (EtOAc/Pen 70:30), as a colorless solid (m.p. $\left.85-88^{\circ} \mathrm{C}\right)$ in $20 \%$ yield. ${ }^{1} \mathrm{H}$ NMR $\left(\mathrm{CDCl}_{3}\right): \delta=7.28-7.22(\mathrm{~m}, 2 \mathrm{H})$, $7.21-7.13(\mathrm{~m}, 3 \mathrm{H}), 4.42$ (brs, $1 \mathrm{H}), 4.25-4.05(\mathrm{~m}, 4 \mathrm{H}), 3.86$ (ddq, $J=7.2$, $10.8,24.4 \mathrm{~Hz}, 2 \mathrm{H}), 2.80(\mathrm{dt}, J=3.6,12.0 \mathrm{~Hz}), 2.58(\mathrm{t}, J=10.4 \mathrm{~Hz}), 2.11-$ $2.02(\mathrm{~m}, 1 \mathrm{H}), 2.00-1.86(\mathrm{~m}, 2 \mathrm{H}), 1.70-1.46(\mathrm{~m}, 3 \mathrm{H}), 1.36(\mathrm{t}, J=7.2 \mathrm{~Hz}$, $3 \mathrm{H}), 1.34(\mathrm{t}, J=7.2 \mathrm{~Hz}, 3 \mathrm{H}), 0.81 \mathrm{ppm}(\mathrm{t}, J=7.2 \mathrm{~Hz}, 3 \mathrm{H}) ;{ }^{13} \mathrm{C} \mathrm{NMR}$ $\left(\mathrm{CDCl}_{3}\right): \delta=173.6\left(\mathrm{~d}, J_{\mathrm{CP}}=3.9 \mathrm{~Hz}\right), 142.2,128.6(2 \times), 127.6(2 \times), 127.1$, $70.6\left(\mathrm{~d}, J_{\mathrm{CP}}=4.4 \mathrm{~Hz}\right), 62.7\left(\mathrm{~d}, J_{\mathrm{CP}}=6.6 \mathrm{~Hz}\right), 62.4\left(\mathrm{~d}, J_{\mathrm{CP}}=6.4 \mathrm{~Hz}\right), 60.5$, $57.8\left(\mathrm{~d}, \quad J_{\mathrm{CP}}=16.5 \mathrm{~Hz}\right), 46.1,42.1\left(\mathrm{~d}, J_{\mathrm{CP}}=138.6 \mathrm{~Hz}\right), 32.9\left(\mathrm{~d}, J_{\mathrm{CP}}=\right.$ $15.8 \mathrm{~Hz}), 24.7\left(\mathrm{~d}, J_{\mathrm{CP}}=5.8 \mathrm{~Hz}\right), 16.75(\mathrm{dd}, J=5.8 \mathrm{~Hz}), 16.69(\mathrm{dd}, J=$ $5.9 \mathrm{~Hz}), 14.0 \mathrm{ppm} ;{ }^{31} \mathrm{P} \mathrm{NMR}\left(\mathrm{CDCl}_{3}\right): \delta=31.49 \mathrm{ppm} ; \mathrm{HRMS}: \mathrm{m} / z$ calcd for $\left[\mathrm{C}_{19} \mathrm{H}_{29} \mathrm{O}_{6} \mathrm{PNa}\right]^{+}: 407.1599$; found: 407.1585; the ee was determined by HPLC analysis using a Chiralpak AD column (hexane/iPrOH 90:10): flow rate $1.0 \mathrm{~mL} \mathrm{~min}^{-1} ; \tau_{\text {major }}=10.7 \mathrm{~min}, \tau_{\text {minor }}=9.4 \mathrm{~min}(96 \% e e$, recrystallized to $99 \% e e) ;[\alpha]_{\mathrm{D}}^{\mathrm{RT}}=-15.35\left(c=0.71\right.$ in $\left.\mathrm{CHCl}_{3}\right)$.

(1S,2S,3R,6R)-Ethyl 3-(diethoxyphosphoryl)-2-hydroxy-6-phenylcyclohexanecarboxylate (12 b; major diastereomer): Compound $12 \mathrm{~b}$ was isolated by FC using Iatrobeads (EtOAc/Pen 70:30), as a colorless oil in $75 \%$ yield. ${ }^{1} \mathrm{H}$ NMR $\left(\mathrm{CDCl}_{3}\right): \delta=7.26(\mathrm{t}, J=7.5 \mathrm{~Hz}, 2 \mathrm{H}), 7.19-7.16(\mathrm{~m}$, $3 \mathrm{H}), 4.63(\mathrm{~d}, J=6.5 \mathrm{~Hz}, 1 \mathrm{H}), 4.26-4.11(\mathrm{~m}, 4 \mathrm{H}), 3.93-3.84(\mathrm{~m}, 2 \mathrm{H}), 3.78$ $(\mathrm{s}, 1 \mathrm{H}), 3.23(\mathrm{dt}, J=3.7,12.3 \mathrm{~Hz}, 1 \mathrm{H}), 2.66(\mathrm{dd}, J=2.1,12.1 \mathrm{~Hz}, 1 \mathrm{H})$, 2.17-2.05 (m, 1 H), 2.04-1.93 (m, 2 H), 1.89-1.84 (m, 1 H), 1.50 (ddd, $J=$ 4.0, 13.3, 26.2 Hz, 1 H), $1.35(\mathrm{dt}, J=1.7,7.0 \mathrm{~Hz}, 6 \mathrm{H}), 0.89 \mathrm{ppm}(\mathrm{t}, J=$ $7.1 \mathrm{~Hz}, 3 \mathrm{H}) ;{ }^{13} \mathrm{C} \mathrm{NMR}\left(\mathrm{CDCl}_{3}\right): \delta=173.4\left(\mathrm{~d}, J_{\mathrm{CP}}=4.0 \mathrm{~Hz}\right), 143.6,128.3$ $(2 \times), 127.4(2 \times), 126.5,66.1\left(\mathrm{~d}, J_{\mathrm{CP}}=4.5 \mathrm{~Hz}\right), 62.4\left(\mathrm{~d}, J_{\mathrm{CP}}=6.5 \mathrm{~Hz}\right), 61.7$ $\left(\mathrm{d}, J_{\mathrm{CP}}=6.8 \mathrm{~Hz}\right), 60.5,53.3\left(\mathrm{~d}, J_{\mathrm{CP}}=15.8 \mathrm{~Hz}\right), 40.2\left(\mathrm{~d}, J_{\mathrm{CP}}=142.0 \mathrm{~Hz}\right)$, $39.4,33.9\left(\mathrm{~d}, J_{\mathrm{CP}}=16.9 \mathrm{~Hz}\right), 20.1\left(\mathrm{~d}, J_{\mathrm{CP}}=2.9 \mathrm{~Hz}\right), 16.5\left(\mathrm{~d}, J_{\mathrm{CP}}=6.0 \mathrm{~Hz}\right.$, $2 \times), 13.7 \mathrm{ppm} ;{ }^{31} \mathrm{P}$ NMR $\left(\mathrm{CDCl}_{3}\right): \delta=31.41 \mathrm{ppm}$; HRMS : $\mathrm{m} / z$ calcd for $\left[\mathrm{C}_{19} \mathrm{H}_{29} \mathrm{O}_{6} \mathrm{PNa}\right]^{+}: 407.1599$; found: 407.1585 ; the ee was determined by HPLC analysis using a Chiralpak AD column (hexane/iPrOH 90:10): flow rate $1.0 \mathrm{~mL} \mathrm{~min}^{-1} ; \tau_{\text {major }}=10.7 \mathrm{~min}, \tau_{\text {minor }}=9.4 \mathrm{~min}(96 \% e e) ;[\alpha]_{\mathrm{D}}^{\mathrm{RT}}=$ $-2.18\left(c=0.79\right.$ in $\left.\mathrm{CHCl}_{3}\right)$.

Preparation of ethyl 2-hydroxy-3-methylene-6-phenylcyclohex-1-enecarboxylate (16a): $\mathrm{Pd} / \mathrm{C}(10 \mathrm{~mol} \%, 52.0 \mathrm{mg}, 0.048 \mathrm{mmol})$ was added to a solution of $\mathbf{3 a}(76 \mathrm{mg}, 0.2 \mathrm{mmol})$ in EtOAc $(5 \mathrm{~mL})$, and the resulting mixture was stirred under $\mathrm{H}_{2}$ atmosphere for $90 \mathrm{~min}$. Then the mixture was filtered through Celite and concentrated in vacuo. The residue was dissolved in THF (1 mL), and formaldehyde (aq $37 \%, 0.180 \mathrm{~mL}, 2.4 \mathrm{mmol}$ ) and a solution of $\mathrm{K}_{2} \mathrm{CO}_{3}(83 \mathrm{mg}, 0.6 \mathrm{mmol})$ in $\mathrm{H}_{2} \mathrm{O}(1 \mathrm{~mL})$ were added to this solution. The resulting mixture was stirred at RT overnight and then quenched with brine $(10 \mathrm{~mL})$ and extracted with $\mathrm{CH}_{2} \mathrm{Cl}_{2}(3 \times 15 \mathrm{~mL})$. The combined organic layers were dried over $\mathrm{MgSO}_{4}$ and evaporated. Compound 16a was isolated by $\mathrm{FC}$ using Iatrobeads $\left(\mathrm{DCM} / \mathrm{Et}_{2} \mathrm{O} 4: 1\right)$, as a white solid (m.p. $63-66^{\circ} \mathrm{C}$ ) in $39 \%$ yield (over 2 steps). ${ }^{1} \mathrm{H}$ NMR $\left(\mathrm{CDCl}_{3}\right): \delta=12.43(\mathrm{~s}, 1 \mathrm{H}), 7.27(\mathrm{~d}, J=7.3 \mathrm{~Hz}, 2 \mathrm{H}), 7.19-7.15(\mathrm{~m}, 3 \mathrm{H})$, $5.96(\mathrm{~s}, 1 \mathrm{H}), 5.26(\mathrm{~s}, 1 \mathrm{H}), 4.10-4.00(\mathrm{~m}, 3 \mathrm{H}), 2.42-2.36(\mathrm{~m}, 1 \mathrm{H}), 1.98(\mathrm{dt}$, $J=3.6,15.0 \mathrm{~Hz}, 1 \mathrm{H}), 2.08-2.00(\mathrm{~m}, 1 \mathrm{H}), 1.80-1.76(\mathrm{~m}, 1 \mathrm{H}), 1.00 \mathrm{ppm}(\mathrm{t}$, $J=7.2 \mathrm{~Hz}, 3 \mathrm{H}) ;{ }^{13} \mathrm{C} \mathrm{NMR}\left(\mathrm{CDCl}_{3}\right): \delta=172.7,165.2,144.8,138.2,128.0$ $(2 \times), 127.6(2 \times), 125.9,116.6,110.9,60.5,39.4,31.0,26.1,13.8 \mathrm{ppm}$; HRMS: $m / z$ calcd for $\left[\mathrm{C}_{16} \mathrm{H}_{18} \mathrm{O}_{3} \mathrm{Na}\right]^{+}: 281.1154$; found: 281.1158 ; the $e e$ was determined by HPLC analysis using a Chiralcel OJ column (hexane/ $i \operatorname{PrOH}$ 93:7): flow rate $1.0 \mathrm{~mL} \mathrm{~min}^{-1} ; \tau_{\text {major }}=4.3 \mathrm{~min}, \tau_{\text {minor }}=4.9 \mathrm{~min}$ $(97 \% e e) ;[\alpha]_{\mathrm{D}}^{\mathrm{RT}}=+58.21\left(c=0.52\right.$ in $\left.\mathrm{CHCl}_{3}\right)$.

Preparation of ethyl 2-hydroxy-3-methylene-4,6-diphenylcyclohex-1-enecarboxylate (16b): A solution of $\mathbf{3 a}(75 \mathrm{mg}, 0.197 \mathrm{mmol})$ in THF $(2 \mathrm{~mL})$ was added dropwise to a solution of $\mathrm{CuI}(46.0 \mathrm{mg}, 0.24 \mathrm{mmol})$ and $\mathrm{PhMgBr}(1 \mathrm{M}$ in THF, $0.24 \mathrm{~mL}, 0.24 \mathrm{mmol})$ in THF $(2 \mathrm{~mL})$ at $0^{\circ} \mathrm{C}$. The reaction was stirred at this temperature for $1 \mathrm{~h}$. The mixture was acidified with $1 \mathrm{M} \mathrm{HCl}(8 \mathrm{~mL})$ and extracted with $\mathrm{CH}_{2} \mathrm{Cl}_{2}(4 \times 10 \mathrm{~mL})$. The combined organic layers were washed with brine, dried $\left(\mathrm{MgSO}_{4}\right)$, and concentrated in vacuo. The residue was dissolved in THF $(1 \mathrm{~mL})$, and formaldehyde (aq $37 \%, 0.180 \mathrm{~mL}, 2.4 \mathrm{mmol})$ and a solution of $\mathrm{K}_{2} \mathrm{CO}_{3}(83 \mathrm{mg}$, $0.6 \mathrm{mmol})$ in $\mathrm{H}_{2} \mathrm{O}(1 \mathrm{~mL})$ were added to this solution. The resulting mixture was stirred at RT overnight and then quenched with aqueous sat. $\mathrm{NaCl}$ solution $(10 \mathrm{~mL})$ and extracted with $\mathrm{CH}_{2} \mathrm{Cl}_{2}(3 \times 15 \mathrm{~mL})$. The combined organic layers were dried over $\mathrm{MgSO}_{4}$ and concentrated in vacuo. Compound $\mathbf{1 6 b}$ was isolated by $\mathrm{FC}$ using Iatrobeads $\left(\mathrm{CH}_{2} \mathrm{Cl}_{2} / \mathrm{Et}_{2} \mathrm{O} 4: 1\right)$, as a colorless oil in $44 \%$ yield (over 2 steps) as a mixture of diastereoisomers (>95:5). Major diastereoisomer: ${ }^{1} \mathrm{H} \mathrm{NMR}\left(\mathrm{CDCl}_{3}\right): \delta=12.59$ (s, $1 \mathrm{H}), 7.31-7.26(\mathrm{~m}, 4 \mathrm{H}), 7.23-7.19(\mathrm{~m}, 4 \mathrm{H}), 7.11(\mathrm{dd}, J=1.5,8.0 \mathrm{~Hz}, 2 \mathrm{H})$, $6.15(\mathrm{~s}, 1 \mathrm{H}), 4.89-4.88(\mathrm{~m}, 1 \mathrm{H}), 4.12-4.05(\mathrm{~m}, 3 \mathrm{H}), 3.55$ (ddd, $J=2.4,5.7$, $12.5 \mathrm{~Hz}, 1 \mathrm{H}), 2.40(\mathrm{dt}, J=5.4,12.9 \mathrm{~Hz}, 1 \mathrm{H}), 1.98(\mathrm{dt}, J=3.4,12.9 \mathrm{~Hz}$, $1 \mathrm{H}), 1.02 \mathrm{ppm}(\mathrm{t}, J=7.1 \mathrm{~Hz}, 3 \mathrm{H}) ;{ }^{13} \mathrm{C} \mathrm{NMR}\left(\mathrm{CDCl}_{3}\right): \delta=172.7,165.3$, 144.4, 143.1, 142.3, $128.5(2 \times), 128.4(2 \times), 128.1(2 \times), 127.6(2 \times), 126.6$, 126, 119.1, 101.4, 60.6, 41.1, 38.6, 38.5, $13.9 \mathrm{ppm}$; minor diastereoisomer: ${ }^{1} \mathrm{H}$ NMR (400 MHz, $\mathrm{CDCl}_{3}$ ): $\delta=12.39$ (s, 1 H), 5.91-5.90 (s, 1H), 4.51- 
$4.49(\mathrm{~m}, 1 \mathrm{H}), 1.05 \mathrm{ppm}(\mathrm{t}, J=7.1 \mathrm{~Hz}, 3 \mathrm{H})$; HRMS: $\mathrm{m} / \mathrm{z}$ calcd for $\left[\mathrm{C}_{22} \mathrm{H}_{22} \mathrm{O}_{3} \mathrm{Na}\right]^{+}$: 357.1467 ; found: 357.1461 ; the $e e$ was determined by HPLC analysis using a Chiralcel OJ column (hexane/iPrOH 90:10): flow rate $1.0 \mathrm{~mL} \mathrm{~min}^{-1} ; \tau_{\text {major }}=4.6 \mathrm{~min}, \tau_{\text {minor }}=5.2 \mathrm{~min}(96 \% e e) ; \quad[\alpha]_{\mathrm{D}}^{\mathrm{RT}}=$ $+38.21\left(c=0.78\right.$ in $\left.\mathrm{CHCl}_{3}\right)$.

\section{Acknowledgements}

This work was made possible by a grant from Danish National Research Foundation and OChemSchool.

[1] For recent reviews on organocatalysis, see for example: a) P. L. Dalko, L. Moisan, Angew. Chem. 2004, 116, 5248; Angew. Chem. Int. Ed. 2004, 43, 5138; b) A. Berkessel, H. Gröger, Asymmetric Organocatalysis, Wiley-VCH, Weinheim, 2004; c) special issue on organocatalysis: Acc. Chem. Res. 2004, 37, 487; d) J. Seayad, B. List, Org. Biomol. Chem. 2005, 3, 719; e) B. List, J.-W. Yang, Science 2006, 313, 1584; f) B. List, Chem. Commun. 2006, 819; g) M. J. Gaunt, C. C. C. Johansson, A. McNally, N. C. Vo, Drug Discovery Today 2007, 12, 8; h) P. I. Dalko, Enantioselective Organocatalysis, Wiley$\mathrm{VCH}$, Weinheim, 2007; i) special issue on organocatalysis: Chem. Rev. 2007, 107, 5413; j) A. Dondoni, A. Massi, Angew. Chem. 2008, 120, 4716; Angew. Chem. Int. Ed. 2008, 47, 4638; k) P. Melchiorre, M. Marigo, A. Carlone, G. Bartoli, Angew. Chem. 2008, 120, 6232; Angew. Chem. Int. Ed. 2008, 47, 6138.

[2] For recent reviews of $\alpha$-functionalizations using enamine activation, see for example: a) M. Marigo, K. A. Jørgensen, Chem. Commun. 2006, 2001; b) G. Guillena, D. J. Ramón, Tetrahedron: Asymmetry 2006, 17, 1465; c) S. Bertelsen, M. Nielsen, K. A. Jørgensen, Angew. Chem. 2007, 119, 7500; Angew. Chem. Int. Ed. 2007, 46, 7356; for examples see: d) B. List, R. A. Lerner, C. F. Barbas, J. Am. Chem. Soc. 2000, 122, 2395; e) M. P. Brochu, S. P. Brown, D. W. C. MacMillan, J. Am. Chem. Soc. 2004, 126, 4108; f) M. T. Hechavarria Fonseca, B. List, Angew. Chem. 2004, 116, 4048; Angew. Chem. Int. Ed. 2004, 43, 3958; g) J. Alemán, S. Cabrera, E. Maerten, J. Overgaard, K. A. Jørgensen, Angew. Chem. 2007, 119, 5616; Angew. Chem. Int. Ed. 2007, 46, 5520; h) M. Tiecco, A. Carlone, S. Sternativo, F. Marini, G. Bartoli, P. Melchiorre, Angew. Chem. 2007, 119, 7006; Angew. Chem. Int. Ed. 2007, 46, 6882; i) C. Palomo, S. Vera, I. Velilla, A. Mielgo, E. Gómez-Bengoa, Angew. Chem. 2007, 119, 8200; Angew. Chem. Int. Ed. 2007, 46, 8054; j) S. Sulzer-Mossé, M. Tissot, A. Alexakis, Org. Lett. 2007, 9, 3749; k) J. Carpenter, A. B. Northrup, dM. Chung, J. J. M. Wiener, S.-G. Kim, D. W. C. MacMillan, Angew. Chem. 2008, 120, 3624; Angew. Chem. Int. Ed. 2008, 47, 3568; 1) £. Albrecht, B. Richter, H. Krawczyk, K. A. Jørgensen, J. Org. Chem. 2008, 73,8337 .

[3] For recent reviews on conjugated additions using iminium-ion activation, see: a) G. Lelais, D. W. C. MacMillan, Aldrichimica Acta 2006, 39, 79; b) S. Sulzer-Mossé, A. Alexakis, Chem. Commun. 2007, 3123; c) S. B. Tsogoeva, Eur. J. Org. Chem. 2007, 1701; d) D. Almasi, D. A. Alonso, C. Najera, Tetrahedron: Asymmetry 2007, 18, 299; e) J. L. Vicario, D. Badía, L. Carrillo, Synthesis 2007, 2065; for examples, see: f) K. A. Ahrendt, C. J. Borths, D. W. C. MacMillan, J. Am. Chem. Soc. 2000, 122, 4243; g) S. P. Brown, N. C. Goodwin, D. W. C. MacMillan, J. Am. Chem. Soc. 2003, 125, 1192; h) S. Brandau, A. Landa, J. Franzén, M. Marigo, K. A. Jørgensen, Angew. Chem. 2006, 118, 4411; Angew. Chem. Int. Ed. 2006, 45, 4305; i) H. Gotoh, R. Masui, H. Ogino, M. Shoji, Y. Hayashi, Angew. Chem. 2006, 118, 7007; Angew. Chem. Int. Ed. 2006, 45, 6853; j) S. Mayer, B. List, Angew. Chem. 2006, 118, 4299; Angew. Chem. Int. Ed. 2006, 45, 4193; k) R. Rios, H. Sundén, I. Ibrahem, G. Zhao, L. Eriksson, A. Córdova, Tetrahedron Lett. 2006, 47, 8547; 1) A. Carlone, M. Marigo, C. North, A. Landa, K. A. Jørgensen, Chem. Commun. 2006, 4928; m) Y. K. Chen, M. Yoshida, D. W. C. MacMillan, J. Am. Chem. Soc. 2006, 128, 9328; n) S. Bertelsen, P. Dinér, R. L. Johansen, K. A. Jør- gensen, J. Am. Chem. Soc. 2007, 129, 1536; o) P. Dinér, M. Nielsen, M. Marigo, K. A. Jørgensen, Angew. Chem. 2007, 119, 2029; Angew. Chem. Int. Ed. 2007, 46, 1983; p) A. Carlone, G. Bartoli, M. Bosco, L. Sambri, P. Melchiorre, Angew. Chem. 2007, 119, 4588; Angew. Chem. Int. Ed. 2007, 46, 4504; q) I. Ibrahem, R. Rios, J. Vesely, P. Hammar, L. Eriksson, F. Himo, A Córdova, Angew. Chem. 2007, 119, 4591; Angew. Chem. Int. Ed. 2007, 46, 4507; r) E. Maerten, S Cabrera, A. Kjaersgaard, K. A. Jørgensen, J. Org. Chem. 2007, 72, 8893 ; s) S. Bertelsen, R. L. Johansen, K. A. Jørgensen, Chem. Commun. 2008, 3016; t) P. Bolze, G. Dickmeiss, K. A. Jørgensen, Org. Lett. 2008, 10, 3753; u) S. Cabrera, E. Reyes, J. Alemán, A Milelli, S, Kobbelgaard, K. A. Jørgensen, J. Am. Chem. Soc. 2008, 130, 12031.

[4] For nomenclature regarding multicomponent reactions, see: a) C. J. Chapman, C. G. Frost, Synthesis 2007, 1 and references therein; for recent reviews on multicomponent reactions see: b) D. Tejedor, D Gonzalez-Cruz, A. Santos-Expositto, J. J. Marrero-Tellado, P. de Armas, F. Garcia-Tellado, Chem. Eur. J. 2005, 11, 3502; c) D. J. Ramón, M. Yus, Angew. Chem. 2005, 117, 1628; Angew. Chem. Int. Ed. 2005 , 44, 1602; d) F. Liéby-Muller, C. Simon, T. Constantieux, J. Rodriguez, OSAR Comb. Sci. 2006, 25, 432; e) H. Guo, J. Ma, Angew. Chem. 2006, 118, 362; Angew. Chem. Int. Ed. 2006, 45, 354; f) D. Enders, C. Grondal, M. R. M. Hüttl, Angew. Chem. 2007, 119, 1590; Angew. Chem. Int. Ed. 2007, 46, 1570; g) G. Guillena, D. J. Ramón, M. Yus, Tetrahedron: Asymmetry 2007, 18, 693; h) X. Yu, W. Wang, Org. Biomol. Chem. 2008, 6, 2037; for some selected examples see: i) Y. Yamamoto, N. Momiyama, H. Yamamoto, J. Am. Chem. Soc 2004, 126, 5962; j) D. Enders, M. R. M. Hüttl, C. Grondal, G. Raabe, Nature 2006, 441, 861; k) D. Enders, M. R. M. Hüttl, J. Runsink, G. Raabe, B. Wendt, Angew. Chem. 2007, 119, 471; Angew. Chem. Int Ed. 2007, 46, 467; 1) Y. Hayashi, T. Okano, S. Aratake, D. Hazelard Angew. Chem. 2007, 119, 5010; Angew. Chem. Int. Ed. 2007, 46, 4922; m) J. Zhou, B. List, J. Am. Chem. Soc. 2007, 129, 7498; n) D. Enders, C. Wang, J. W. Bats, Angew. Chem. 2008, 120, 7649; Angew. Chem. Int. Ed. 2008, 47, 7539.

[5] a) N. Halland, P. S. Aburel, K. A. Jørgensen, Angew. Chem. 2004 116, 1292; Angew. Chem. Int. Ed. 2004, 43, 1272; b) J. Pulkkinen, P. S. Aburel, N. Halland, K. A. Jørgensen, Adv. Synth. Catal. 2004, 346, 1077; c) M. Marigo, T. Schulte, J. Franzén, K. A. Jørgensen, J. Am. Chem. Soc. 2005, 127, 15710; d) M. Marigo, J. Franzén, T. B. Poulsen, W. Zhuang, K. A. Jørgensen, J. Am. Chem. Soc. 2005, 127 6964; e) S. Brandau, E. Maerten, K. A. Jørgensen, J. Am. Chem. Soc. 2006, 128, 14986; f) M. Marigo, S. Bertelsen, A. Landa, K. A Jørgensen, J. Am. Chem. Soc. 2006, 128, 5475; g) A. Carlone, S. Cabrera, M. Marigo, K. A. Jørgensen, Angew. Chem. 2007, 119, 1119 Angew. Chem. Int. Ed. 2007, 46, 1101; h) E. Reyes, H. Jiang, A Milelli, P. Elsner, R. G. Hazell, K. A. Jørgensen, Angew. Chem. 2007, 119, 9362; Angew. Chem. Int. Ed. 2007, 46, 9202; i) P. T. Franke, R. L. Johansen, S. Bertelsen, K. A. Jørgensen, Chem. Asian J. 2008, 3, 216; j) S. Cabrera, J. Alemán, P. Bolze, S. Bertelsen, K. A Jørgensen, Angew. Chem. 2008, 120, 127; Angew. Chem. Int. Ed. 2008, 47, 121; k) P. T. Franke, B. Richter, K. A. Jørgensen, Chem Eur. J. 2008, 14, 6317 .

[6] For examples of organocatalytic one-pot multicomponent reactions involving iminium-catalyzed Michael addition as a first step, see: a) J. F. Austin, S.-G. Kim, C. J. Sinz, W.-J. Hiao, D. W. C. MacMillan, Proc. Natl. Acad. Sci. USA 2004, 101, 5482; b) R. K. Kunz, D. W. C. MacMillan, J. Am. Chem. Soc. 2005, 127, 3240; c) J. W. Yang, M. T. Hechavarria Fonseca, B. List, J. Am. Chem. Soc. 2005, 127, 15036; d) Y. Huang, A. M. Walji, C. H. Larsen, D. W. C. MacMillan, J. Am Chem. Soc. 2005, 127, 15051; e) D. Gryko, Tetrahedron: Asymmetry 2005, 16, 1377; f) R. Rios, H. Sundén, J. Vesely, G.-L. Zhao, P. Dziedzic, A. Córdova, Adv. Synth. Catal. 2007, 349, 1028; g) J Vesely, I. Ibrahem, G.-L. Zhao, R. Rios, A. Córdova, Angew. Chem. 2007, 119, 792; Angew. Chem. Int. Ed. 2007, 46, 778; h) L. Zu, H. Li, H. Xie, J. Wang, W. Jiang, Y. Tang, W. Wang, Angew. Chem. 2007, 119, 3806; Angew. Chem. Int. Ed. 2007, 46, 3732; i) H. M. Hansen, D. A. Longbottom, S. V. Ley, Chem. Commun. 2006, 4838; j) W. Wang, H. Li, J. Wang, L. Zu, J. Am. Chem. Soc. 2006, 128, 10354; 
k) B.-C. Hong, M.-F. Wu, H.-C. Tseng, J.-H. Liao, Org. Lett. 2006, 8 , 2217; 1) T. Govender, L. Hojabri, F. M. Maghaddam, P. I. Arvidsson, Tetrahedron: Asymmetry 2006, 17, 1763; m) R. Rios, H. Sundén, I. Ibrahem, G.-L. Zhao, A. Córdova, Tetrahedron Lett. 2006, 47, 8679; n) H. Sundén, R. Rios, I. Ibrahem, G.-L. Zhao, L. Eriksson, A. Córdova, Adv. Synth. Catal. 2007, 349, 827; o) J. Wang, H. Li, H. Xie, L. $\mathrm{Zu}, \mathrm{X}$. Shen, W. Wang, Angew. Chem. 2007, 119, 9208; Angew. Chem. Int. Ed. 2007, 46, 9050; p) H. Li, J. Wang, T. E-Nunu, L.-S. Zu, W. Jiang, S.-H. Wei, W. Wang, Chem. Commun. 2007, 507; q) I. Ibrahem, R. Rios, J. Vesely, G.-L. Zhao, A. Córdova, Chem. Commun. 2007, 849; r) H. Sundén, I. Ibrahem, G.-L. Zhao, L. Eriksson, A. Córdova, Chem. Eur. J. 2007, 13, 574; s) H. Xie, L. Zu, H. Li, J. Wang, W. Wang, J. Am. Chem. Soc. 2007, 129, 10886; t) H. Li, J. Wang, H.-X. Xie, L.-S. Zu, W. Jiang, E. N. Duesler, W. Wang, Org. Lett. 2007, 9, 965; u) H. Li, L. Zu, H. Xie, J. Wang, W. Jiang, W. Wang, Org. Lett. 2007, 9, 1833; v) D. Enders, A. A. Narine, T. R. Benninghaus, G. Raabe, Synlett 2007, 1667; w) R. Rios, J. Vesely, H. Sundén, I. Ibrahem, G.-L. Zhao, A. Córdova, Tetrahedron Lett. 2007, 48, 5835; x) M.-K. Zhu, Q. Wie, L.-Z. Gong, Adv. Synth. Catal. 2008, 350, 1281; y) G.-L. Zhao, R. Rios, J. Vesely, L. Eriksson, A. Córdova, Angew. Chem. 2008, 120, 8596; Angew. Chem. Int. Ed. 2008, 47, 8468; z) M. Rueping, E. Sugiono, E. Merino, Chem. Eur. J. 2008, 14, 6329; aa) I. Ibrahem, G.-L. Zhao, R. Rios, J. Vesely, H. Sundén, P. Dziedzic, A. Córdova, Chem. Eur. J. 2008, 14, 7867.

[7] For some selected examples see: a) J. M. Llera, B. Fraser-Reid, $J$. Org. Chem. 1989, 54, 5544; b) T. Kan, S. Hosokawa, S. Nara, M. Oikawa, S. Ito, F. Matsuda, H. Shirahama, J. Org. Chem. 1994, 59, 5532; c) S. G. Davies, G. Bhalay, Tetrahedron: Asymmetry 1996, 7 , 1595; d) S. Hanessian, A. M. Griffin, M. J. Rozema, Bioorg. Med. Chem. Lett. 1997, 7, 1857; e) K. Yamamoto, M. F. Hentemann, J. G. Allen, S. J. Danishefsky, Chem. Eur. J. 2003, 9, 3242; f) S. Vargas, A.
Suarez, E. Alvarez, A. Pizzano, Chem. Eur. J. 2008, 14, 9856, and references therein.

[8] For some selected examples see: a) R. L. Danheiser, H. Sard, J. Org Chem. 1980, 45, 4810; b) K. M. Pietrusiewicz, J. Monkiewicz, R. Bodalski, J. Org. Chem. 1983, 48, 788; c) K. M. Pietrusiewicz, I. Salamoñczyk, J. Org. Chem. 1988, 53, 2837; d) J. K. F. Geirsson, J. F. Johannesdottir, J. Org. Chem. 1996, 61, 7320; e) J. L. Loebach, D. M. Bennett, R. L. Danheiser, J. Org. Chem. 1998, 63, 8380; f) T. K. M. Shing, X. Y. Zhu, Y. Y. Yeung, Chem. Eur. J. 2003, 9, 5489.

[9] For the first application of silyldiarylprolinol ethers as catalysts see: a) M. Marigo, T. C. Wabnitz, D. Fielenbach, K. A. Jørgensen, Angew. Chem. 2005, 117, 804; Angew. Chem. Int. Ed. 2005, 44, 794. See also: b) M. Marigo, D. Fielenbach, A. Braunton, A. Kjærsgaard, K. A. Jørgensen, Angew. Chem. 2005, 117, 3769; Angew. Chem. Int Ed. 2005, 44, 3703; c) Y. Hayashi, H. Gotoh, T. Hayashi, M. Shoji, Angew. Chem. 2005, 117, 4284; Angew. Chem. Int. Ed. 2005, 44, 4212; d) J. Franzén, M. Marigo, D. Fielenbach, T. C. Wabnitz, A. Kjærsgaard, K. A. Jørgensen, J. Am. Chem. Soc. 2005, 127, 18296; e) C. Palomo, A. Mielgo, Angew. Chem. 2006, 118, 8042; Angew. Chem. Int. Ed. 2006, 45, 7876; f) A. Mielgo, C. Palomo, Chem. Asian J. 2008, 3, 922.

[10] CCDC-708143 (12a) contains the supplementary crystallographic data for this paper. These data can be obtained free of charge from The Cambridge Crystallographic Data Centre via www.ccdc.cam. ac.uk/data_request/cif.

[11] a) R. Bodalski, K. M. Pietrusiewicz, J. Monkiewicz, J. Koszuk, Tetrahedron Lett. 1980, 21, 2287; b) J.-F. Lavallée, C. Spino, R. Ruel, K. T. Hogan, P. Deslongchamps, Can. J. Chem. 1992, 70, 1406.

Received: November 4, 2008 Published online: January 28, 2009 